Pacific

Journal of

Mathematics

ESSENTIAL DIMENSION AND ERROR-CORRECTING CODES

SHANE CERNELE AND ZinOVy REICHSTEIN

APPENDIX BY ATHENA NGUYEN

Volume 279 No. 1-2

December 2015 


\title{
ESSENTIAL DIMENSION AND ERROR-CORRECTING CODES
}

\author{
Shane CeRnele And Zinovy Reichstein \\ APPENDiX By ATHENA NGUYEN \\ To the memory of Robert Steinberg
}

One of the important open problems in the theory of central simple algebras is to compute the essential dimension of $\mathbf{G L}_{n} / \mu_{m}$, i.e., the essential dimension of a generic division algebra of degree $n$ and exponent dividing $m$. In this paper we study the essential dimension of groups of the form

$$
G=\left(G_{n_{1}} \times \cdots \times G_{n_{r}}\right) / C,
$$

where $C$ is a central subgroup of $\mathrm{GL}_{n_{1}} \times \cdots \times \mathrm{GL}_{n_{r}}$. Equivalently, we are interested in the essential dimension of a generic $r$-tuple $\left(A_{1}, \ldots, A_{r}\right)$ of central simple algebras such that $\operatorname{deg}\left(A_{i}\right)=n_{i}$ and the Brauer classes of $A_{1}, \ldots, A_{r}$ satisfy a system of homogeneous linear equations in the Brauer group. The equations depend on the choice of $C$ via the error-correcting code $\operatorname{Code}(C)$ which we naturally associate to $C$. We focus on the case where $n_{1}, \ldots, n_{r}$ are powers of the same prime. The upper and lower bounds on ed $(G)$ we obtain are expressed in terms of coding-theoretic parameters of $\operatorname{Code}(C)$, such as its weight distribution. Surprisingly, for many groups of the above form the essential dimension becomes easier to estimate when $r \geq 3$; in some cases we even compute the exact value. The Appendix by Athena Nguyen contains an explicit description of the Galois cohomology of groups of the form $\left(\mathrm{GL}_{n_{1}} \times \cdots \times \mathrm{GL}_{n_{r}}\right) / C$. This description and its corollaries are used throughout the paper.

\section{Introduction}

Let $k$ be a base field. Unless otherwise specified, we will assume that every field appearing in this paper contains $k$ and every homomorphism (i.e., inclusion) of fields restricts to the identity map on $k$.

This paper is based on a portion of Cernele's Ph.D. thesis completed at the University of British Columbia. Cernele and Reichstein gratefully acknowledge financial support from the University of British Columbia and the Natural Sciences and Engineering Research Council of Canada.

MSC2010: primary 20G15, 16K20, 16K50; secondary 94B05.

Keywords: essential dimension, central simple algebra, Brauer group, error-correcting code,

Hamming distance. 
We begin by recalling the definition of the essential dimension of a covariant functor $\mathcal{F}$ from the category of fields to the category of sets. Given a field $K$ and an object $\alpha \in \mathcal{F}(K)$, we will say that $\alpha$ descends to an intermediate field $k \subset K_{0} \subset K$ if $\alpha$ lies in the image of the natural map $\mathcal{F}\left(K_{0}\right) \rightarrow \mathcal{F}(K)$. The essential dimension ed $(\alpha)$ of $\alpha$ is defined as the minimal value of $\operatorname{trdeg}_{k}\left(K_{0}\right)$ such that $\alpha$ descends to a subfield $k \subset K_{0} \subset K$. Given a prime integer $p$, the essential dimension ed ${ }_{p}(\alpha)$ of $\alpha$ at $p$ is defined as the minimal value of $\operatorname{trdeg}_{k}\left(K_{0}\right)$, where the minimum is taken over all finite field extensions $L / K$ and all intermediate fields $k \subset K_{0} \subset L$, such that $[L: K]$ is prime to $p$ and $\alpha_{L}$ descends to $K_{0}$.

The essential dimension ed $(\mathcal{F})$ (respectively, the essential dimension $\operatorname{ed}_{p}(\mathcal{F})$ at $p$ ) of the functor $\mathcal{F}$ is defined as the maximal value of ed( $\alpha$ ) (respectively of $\operatorname{ed}_{p}(\alpha)$ ), where the maximum is taken over all field extensions $K / k$ and all objects $\alpha \in \mathcal{F}(K)$.

Informally speaking, $\operatorname{ed}(\alpha)$ is the minimal number of independent parameters required to define $\alpha$, ed $(\mathcal{F})$ is the minimal number of independent parameters required to define any object in $\mathcal{F}$, and $\operatorname{ed}_{p}(\alpha), \operatorname{ed}_{p}(\mathcal{F})$ are relative versions of these notions at a prime $p$. These relative versions are somewhat less intuitive, but they tend to be more accessible and more amenable to computation than $\operatorname{ed}(\alpha)$ and $\operatorname{ed}(\mathcal{F})$. Clearly $\operatorname{ed}(\alpha) \geqslant \operatorname{ed}_{p}(\alpha)$ for each $\alpha$, and $\operatorname{ed}(\mathcal{F}) \geqslant \operatorname{ed}_{p}(\mathcal{F})$. In most cases of interest, $\operatorname{ed}(\alpha)$ is finite for every $\alpha$. On the other hand, ed( $\mathcal{F})$ (and even $\operatorname{ed}_{p}(\mathcal{F})$ ) can be infinite. For an introduction to the theory of essential dimension, we refer the reader to the surveys [Berhuy and Favi 2003; Reichstein 2010; 2012; Merkurjev 2013].

To every algebraic group $G$ one can associate the functor

$\mathcal{F}_{G}:=H^{1}(*, G): K \mapsto\{$ isomorphism classes of $G$-torsors over $\operatorname{Spec}(K)\}$.

If $G$ is affine, then the essential dimension of this functor is known to be finite; it is usually denoted by $\operatorname{ed}(G)$, rather than $\operatorname{ed}\left(\mathcal{F}_{G}\right)$. For many specific groups $G$, $H^{1}(K, G)$ is in a natural bijective correspondence with the set of isomorphism classes of some algebraic objects defined over $K$. In such cases, $\operatorname{ed}(G)$ may be viewed as the minimal number of independent parameters required to define any object of this type. This number is often related to classical problems in algebra.

For example, in the case where $G$ is the projective linear group $\mathrm{PGL}_{n}$, the objects in question are central simple algebras. That is,

\section{$H^{1}\left(K, \mathrm{PGL}_{n}\right)=\{$ isomorphism classes of}

central simple $K$-algebras of degree $n$ \}.

The problem of computing ed $\left(\mathrm{PGL}_{n}\right)$ is one of the important open problems in the theory of central simple algebras; see [Auel et al. 2011, Section 6]. This problem was first posed by C. Procesi, who showed (using different terminology) that

$$
\operatorname{ed}\left(\mathrm{PGL}_{n}\right) \leqslant n^{2}
$$


see [Procesi 1967, Theorem 2.1]. Stronger (but still quadratic) upper bounds can be found in [Lorenz et al. 2003, Theorem 1.1] and [Lemire 2004, Theorem 1.6].

A more general but closely related problem is computing ed $\left(\mathrm{GL}_{n} / \mu_{m}\right)$, where $m$ and $n$ are positive integers and $m$ divides $n$. Note that

$$
\begin{array}{r}
H^{1}\left(K, \mathrm{GL}_{n} / \mu_{m}\right)=\{\text { isomorphism classes of central simple } K \text {-algebras } \\
\text { of degree } n \text { and exponent dividing } m\} .
\end{array}
$$

In particular, ed $\left(\mathrm{PGL}_{n}\right)=\mathrm{ed}\left(\mathrm{GL}_{n} / \mu_{n}\right)$. The problem of computing ed $\left(\mathrm{GL}_{n} / \mu_{m}\right)$ partially reduces to the case where $m=p^{s}$ and $n=p^{a}$ are powers of the same prime $p$ and $1 \leqslant s \leqslant a$.

From now on we will always assume that $\operatorname{char}(k) \neq p$. The inequalities

$$
p^{2 a-2}+p^{a-s} \geqslant \operatorname{ed}_{p}\left(\operatorname{GL}_{p^{a}} / \mu_{p^{s}}\right) \geqslant \begin{cases}(a-1) 2^{a-1} & \text { if } p=2 \text { and } s=1, \\ (a-1) p^{a}+p^{a-s} & \text { otherwise, }\end{cases}
$$

proved in [Baek and Merkurjev 2012] represent a striking improvement on the best previously known bounds. (Here $a \geqslant 2$.) Yet the gap between the lower and upper bounds in (4) remains wide. The gap between the best known upper and lower bounds becomes even wider when $\operatorname{ed}_{p}\left(\mathrm{GL}_{p^{a}} / \mu_{p^{s}}\right)$ is replaced by ed(GL $\left.\operatorname{Gl}^{a} / \mu_{p^{s}}\right)$.

These gaps in our understanding of ed(GL $\left.\mathrm{GL}_{n} / \mu_{m}\right)$ will not deter us from considering the vastly more general problem of computing the essential dimension of groups of the form

$$
G:=\left(\mathrm{GL}_{n_{1}} \times \cdots \times \mathrm{GL}_{n_{r}}\right) / C
$$

in the present paper. Here $n_{1}, \ldots, n_{r} \geqslant 2$ are integers, and $C \subset \mathbb{G}_{m}^{r}$ is a central subgroup of $\mathrm{GL}_{n_{1}} \times \cdots \times \mathrm{GL}_{n_{r}}$.

As usual, we will identify elements $\left(m_{1}, \ldots, m_{r}\right)$ of $\mathbb{Z}^{r}$ with characters

$$
x: \mathbb{G}_{m}^{r} \rightarrow \mathbb{G}_{m}, \quad \text { where } \quad x:\left(\tau_{1}, \ldots, \tau_{r}\right) \mapsto \tau_{1}^{m_{1}} \cdots \tau_{r}^{m_{r}} .
$$

The subgroup $C \subset \mathbb{G}_{m}^{r}$ is completely determined by the $\mathbb{Z}$-module

$$
X\left(\mathbb{G}_{m}^{r} / C\right)=\left\{\left(m_{1}, \ldots, m_{r}\right) \in \mathbb{Z}^{r} \mid \tau_{1}^{m_{1}} \cdots \tau_{r}^{m_{r}}=1 \quad \forall\left(\tau_{1}, \ldots, \tau_{r}\right) \in C\right\},
$$

consisting of characters of $\mathbb{G}_{m}^{r}$ which vanish on $C$. The Galois cohomology of $G$ is explicitly described in the Appendix: by Theorem A.1, $H^{1}(K, G)$ is naturally isomorphic to the set of isomorphism classes of $r$-tuples $\left(A_{1}, \ldots, A_{r}\right)$ of central simple $K$-algebras such that

$$
\operatorname{deg}\left(A_{i}\right)=n_{i} \quad \text { and } \quad A_{1}^{\otimes m_{1}} \otimes \cdots \otimes A_{r}^{\otimes m_{r}} \text { is split over } K
$$

for every $\left(m_{1}, \ldots, m_{r}\right) \in X\left(\mathbb{G}_{m}^{r} / C\right)$. (Note that in the special case where $r=1$, we recover (1) and (3).) It follows from this description that the essential dimension 
of $G$ does not change if $C$ is replaced by $C \cap \mu$, where

$$
\mu:=\mu_{n_{1}} \times \cdots \times \mu_{n_{r}} ;
$$

see Corollary A.2. Thus we will assume throughout that $C \subset \mu$. Unless otherwise specified, we will also assume that $n_{1}=p^{a_{1}}, \ldots, n_{r}=p^{a_{r}}$ are powers of the same prime $p$. Here $a_{1}, \ldots, a_{r} \geqslant 1$ are integers. Under these assumptions, instead of $X\left(\mathbb{G}_{m}^{r} / C\right) \subset \mathbb{Z}^{r}$, we will consider the subgroup of

$$
X(\mu)=\left(\mathbb{Z} / p^{a_{1}} \mathbb{Z}\right) \times \cdots \times\left(\mathbb{Z} / p^{a_{r}} \mathbb{Z}\right)
$$

given by

$$
\begin{aligned}
\operatorname{Code}(C):=X & (\mu / C)= \\
& \left\{\left(m_{1}, \ldots, m_{r}\right) \in X(\mu) \mid \tau_{1}^{m_{1}} \cdots \tau_{r}^{m_{r}}=1 \forall\left(\tau_{1}, \ldots, \tau_{r}\right) \in C\right\} .
\end{aligned}
$$

In other words, $\operatorname{Code}(C)$ consists of those characters of $\mu$ which vanish on $C$. The symbol "Code" indicates that we will view this group as an error-correcting code. In particular, we will define the Hamming weight $\mathrm{w}(y)$ of

$$
y=\left(m_{1}, \ldots, m_{r}\right) \in\left(\mathbb{Z} / p^{a_{1}} \mathbb{Z}\right) \times \cdots \times\left(\mathbb{Z} / p^{a_{r}} \mathbb{Z}\right)
$$

as follows. Write $m_{i}:=u_{i} p^{e_{i}}$ with $u_{i} \in\left(\mathbb{Z} / p^{a_{i}} \mathbb{Z}\right)^{*}$ and $0 \leq e_{i} \leq a_{i}$. Then

$$
\mathrm{w}(y):=\sum_{i=1}^{r}\left(a_{i}-e_{i}\right) .
$$

Our main results relate ed $(G)$ to coding-theoretic invariants of $\operatorname{Code}(C)$, such as its weight distribution; see also Corollary A.3. For an introduction to error-correcting coding theory, see [MacWilliams and Sloane 1977].

At this point we should warn the reader that our notions of error-correcting code and Hamming weight are somewhat unusual. In coding-theoretic literature (linear) codes are usually defined as linear subspaces of $\mathbb{F}_{q}^{n}$, where $\mathbb{F}_{q}$ is the field of $q$ elements. In this paper, by a code we will mean an additive subgroup of $\left(\mathbb{Z} / p^{a_{1}} \mathbb{Z}\right) \times \cdots \times\left(\mathbb{Z} / p^{a_{r}} \mathbb{Z}\right)$. Nevertheless, in an important special case, where $a_{1}=\cdots=a_{r}=1$, our codes are linear codes of length $r$ over $\mathbb{F}_{p}$ in the usual sense of error-correcting coding theory, and our definition of the Hamming weight coincides with the usual definition.

Theorem 1.1. Let $p$ be a prime, $G:=\left(\mathrm{GL}_{p^{a_{1}}} \times \cdots \times \mathrm{GL}_{p^{a_{r}}}\right) / C$, where $C \subset$ $\mu_{p^{a_{1}}} \times \cdots \times \mu_{p^{a_{r}}}$ is a central subgroup, and $y_{1}, \ldots, y_{t}$ be a minimal basis for Code $(C)$; see Definition 3.2. Then

(a) $\operatorname{ed}_{p}(G) \geqslant\left(\sum_{i=1}^{t} p^{\mathrm{w}\left(y_{i}\right)}\right)-p^{2 a_{1}}-\cdots-p^{2 a_{r}}+r-t$,

(b) $\operatorname{ed}(G) \leqslant\left(\sum_{i=1}^{t} p^{\mathrm{w}\left(y_{i}\right)}\right)-t+\operatorname{ed}(\bar{G})$ and $\operatorname{ed}_{p}(G) \leqslant\left(\sum_{i=1}^{t} p^{\mathrm{w}\left(y_{i}\right)}\right)-t+\operatorname{ed}_{p}(\bar{G})$, where $\bar{G}:=\mathrm{PGL}_{p^{a_{1}}} \times \cdots \times \mathrm{PGL}_{p^{a}}$. 
Although the upper and lower bounds of Theorem 1.1 never meet, for many central subgroups $C \subset \mu \subset G$, the term $\sum_{i=1}^{t} p^{\mathrm{w}\left(y_{i}\right)}$ is much larger than any of the other terms appearing in the above inequalities and may be viewed as giving the asymptotic value of $\operatorname{ed}(G)$. In particular, note that in view of (2),

$$
\operatorname{ed}_{p}(\bar{G}) \leqslant \operatorname{ed}(\bar{G}) \leqslant \operatorname{ed}\left(\operatorname{PGL}_{p^{a_{1}}}\right)+\cdots+\operatorname{ed}\left(\operatorname{PGL}_{p^{a_{r}}}\right) \leqslant p^{2 a_{1}}+\cdots+p^{2 a_{r}} .
$$

Under additional assumptions on $C$, we will determine the exact value of $\operatorname{ed}(G)$; see Theorem 1.2.

The fact that we can determine $\operatorname{ed}(G)$ for many choices of $C$, either asymptotically or exactly, was rather surprising to us, given the wide gap between the best known upper and lower bounds on $\operatorname{ed}(G)$ in the simplest case, where $r=1$; see (4). Our informal explanation of this surprising phenomenon is as follows. If $\operatorname{Code}(C)$ can be generated by vectors $y_{1}, \ldots, y_{t}$ of small weight, then $\sum_{i=1}^{t} p^{\mathrm{w}\left(y_{i}\right)}$ no longer dominates the other terms. In particular, this always happens if $r \leqslant 2$. In such cases the value of $\operatorname{ed}(G)$ is controlled by the more subtle "lower order effects", which are poorly understood.

To state our next result, we will need the following terminology. Suppose that $2 \leqslant n_{1} \leqslant \cdots \leqslant n_{t}$ and $z=\left(z_{1}, \ldots, z_{r}\right) \in\left(\mathbb{Z} / n_{1} \mathbb{Z}\right) \times \cdots \times\left(\mathbb{Z} / n_{r} \mathbb{Z}\right)$, where $z_{j_{1}}, \ldots, z_{j_{s}} \neq 0$ for some $1 \leqslant j_{1}<\cdots<j_{s} \leqslant r$ and $z_{j}=0$ for any $j \notin\left\{j_{1}, \ldots, j_{r}\right\}$. We will say that $z$ is balanced if

(i) $n_{j_{s}} \leqslant \frac{1}{2} n_{j_{1}} n_{j_{2}} \cdots n_{j_{s-1}}$ and

(ii) $\left(n_{j_{1}}, \ldots, n_{j_{s}}\right) \neq(2,2,2,2),(3,3,3)$ or $(2, n, n)$ for any $n \geqslant 2$.

Note that condition (i) can only hold if $s \geqslant 3$. In particular, $\left(\mathbb{Z} / n_{1} \mathbb{Z}\right) \times \cdots \times\left(\mathbb{Z} / n_{r} \mathbb{Z}\right)$ has no balanced elements if $r \leqslant 2$. In the sequel, we will usually assume that $n_{1}, \ldots, n_{r}$ are powers of the same prime $p$. In this situation, condition (ii) is vacuous, unless $p=2$ or 3 .

Theorem 1.2. Let $p$ be a prime,

$$
G:=\left(\mathrm{GL}_{p^{a_{1}}} \times \cdots \times \mathrm{GL}_{p^{a_{r}}}\right) / C,
$$

where $a_{r} \geqslant \cdots \geqslant a_{1} \geqslant 1$ are integers, and $C$ is a subgroup of $\mu$, as in (7). Assume that the base field $k$ is of characteristic zero and $\operatorname{Code}(C)$ has a minimal basis $y_{i}=\left(y_{i 1}, \ldots, y_{i r}\right), i=1, \ldots, t$ satisfying the following conditions:

(a) $y_{i j}=-1,0$ or 1 in $\mathbb{Z} / p^{a_{j}} \mathbb{Z}$ for every $i=1, \ldots, t$ and $j=1, \ldots, r$.

(b) For every $j=1, \ldots, r$, there exists an $i \in\{1, \ldots, t\}$ such that $y_{i}$ is balanced and $y_{i j} \neq 0$.

Then $\operatorname{ed}(G)=\operatorname{ed}_{p}(G)=\left(\sum_{i=1}^{t} p^{\mathrm{w}\left(y_{i}\right)}\right)-p^{2 a_{1}}-\cdots-p^{2 a_{r}}+r-t$.

Specializing Theorem 1.2 to the case where $\operatorname{Code}(C)$ is generated by the single element $(1, \ldots, 1)$, we obtain the following. 
Theorem 1.3. Let $a_{r} \geqslant a_{r-1} \geqslant \cdots \geqslant a_{1} \geqslant 1$ be integers and $\mathcal{F}$ : Fields $k_{k} \rightarrow$ Sets be the covariant functor where $\mathcal{F}(K)$ is defined as the set of isomorphism classes of $r$-tuples $\left(A_{1}, \ldots, A_{r}\right)$ of central simple $K$-algebras such that $\operatorname{deg}\left(A_{i}\right)=p^{a_{i}}$ for all $i=1, \ldots, r$, and $A_{1} \otimes \cdots \otimes A_{r}$ is split over $K$.

(a) If $a_{r} \geqslant a_{1}+\cdots+a_{r-1}$, then $\operatorname{ed}(\mathcal{F})=\operatorname{ed}\left(\mathrm{PGL}_{p^{a_{1}}} \times \cdots \times \mathrm{PGL}_{p^{a-1}}\right)$ and

$$
\operatorname{ed}_{p}(\mathcal{F})=\operatorname{ed}_{p}\left(\operatorname{PGL}_{p^{a_{1}}} \times \cdots \times \mathrm{PGL}_{p^{a_{r-1}}}\right) .
$$

In particular, $\operatorname{ed}(\mathcal{F}) \leqslant p^{2 a_{1}}+\cdots+p^{2 a_{r-1}}$.

(b) Assume that char $(k)=0, a_{r}<a_{1}+\cdots+a_{r-1}$, and $\left(p^{a_{1}}, \ldots, p^{a_{r}}\right)$ is not of the form $(2,2,2,2),(3,3,3)$ or $\left(2,2^{a}, 2^{a}\right)$ for any $a \geqslant 1$. Then

$$
\operatorname{ed}(\mathcal{F})=\operatorname{ed}_{p}(\mathcal{F})=p^{a_{1}+\cdots+a_{r}}-\sum_{i=1}^{r} p^{2 a_{i}}+r-1 .
$$

(c) If $\left(p^{a_{1}}, \ldots, p^{a_{r}}\right)=(2,2,2)$, then $\operatorname{ed}(\mathcal{F})=\operatorname{ed}_{2}(\mathcal{F})=3$.

Here part (c) treats the smallest of the exceptional cases in part (b). Note that in this case $p=2, r=3$ and $a_{1}=a_{2}=a_{3}=1$. Thus

$$
p^{a_{1}+\cdots+a_{r}}-\sum_{i=1}^{r} p^{2 a_{i}}+r-1=-2,
$$

and formula (10) fails. The values of $\operatorname{ed}(\mathcal{F})$ and $\operatorname{ed}_{p}(\mathcal{F})$ in the other exceptional cases, where $\left(p^{a_{1}}, \ldots, p^{a_{r}}\right)=(2,2,2,2),(3,3,3)$, or $\left(2,2^{a}, 2^{a}\right)$ for some $a \geqslant 2$, remain open.

The results of this paper naturally lead to combinatorial questions, which we believe to be of independent interest but will not address here. For each code (i.e., subgroup) $X \subset\left(\mathbb{Z} / p^{a_{1}} \mathbb{Z}\right) \times \cdots \times\left(\mathbb{Z} / p^{a_{r}} \mathbb{Z}\right)$ of rank $t$, let $\left(w_{1}, \ldots, w_{t}\right)$ be the minimal profile of $X$ with respect to the Hamming weight function, in the sense of Proposition 3.1. That is, $w_{i}=\mathrm{w}\left(y_{i}\right)$, where $y_{1}, \ldots, y_{t}$ is a minimal basis of $X$. Fixing $p, a_{1} \leqslant \cdots \leqslant a_{r}$ and $t$, and letting $X$ range over all possible codes with these parameters:

- What is the lexicographically largest profile $\left(w_{1}, \ldots, w_{t}\right)$ ?

- What is the maximal value of $w_{t}$ ?

- What is the probability that $w_{1}=\cdots=w_{t}$ ?

- What is the maximal value of $p^{w_{1}}+\cdots+p^{w_{t}}$ ?

- What is the average value of $p^{w_{1}}+\cdots+p^{w_{t}}$ ?

- What is the probability that $w_{t}>2 a_{r}$ ? 
Note that the expression $p^{w_{1}}+\cdots+p^{w_{t}}$ appears in the formulas given in Theorem 1.1. For large $p$, the condition that $w_{t}>2 a_{r}$ makes $p^{w_{1}}+\cdots+p^{w_{t}}$ the dominant term in these formulas. To the best of our knowledge, questions of this type (focusing on the minimal profile of a code, rather than the minimal weight) have not been previously investigated by coding theorists, even in the case where $a_{1}=\cdots=a_{r}=1$.

The rest of this paper is structured as follows. In Section 2 we prove general bounds on the essential dimension of certain central extensions of algebraic groups. These bounds will serve as the starting point for the proofs of the main theorems. To make these bounds explicit for groups of the form $\left(\mathrm{GL}_{p^{a_{1}}} \times \cdots \times \mathrm{GL}_{p^{a_{r}}}\right) / C$, we introduce and study the notion of a minimal basis in Section 3. Theorems 1.1, 1.2 and 1.3 are then proved in Sections 4, 5 and 6, respectively. The Appendix by Athena Nguyen contains an explicit description of the Galois cohomology of groups of the form (5). This description and its corollaries are used throughout the paper.

\section{Essential dimension and central extensions}

Let $T=\mathbb{G}_{m}^{r}$ be a split $k$-torus of rank $r$, and

$$
1 \rightarrow T \rightarrow G \rightarrow \bar{G} \rightarrow 1
$$

be a central exact sequence of affine algebraic groups. This sequence gives rise to the exact sequence of pointed sets

$$
H^{1}(K, G) \rightarrow H^{1}(K, \bar{G}) \stackrel{\partial}{\longrightarrow} H^{2}(K, T)
$$

for any field extension $K$ of the base field $k$. Any character $x: T \rightarrow \mathbb{G}_{m}$, induces a homomorphism $x_{*}: H^{2}(K, T) \rightarrow H^{2}\left(K, \mathbb{G}_{m}\right)$. We define ind ${ }^{x}(G, T)$ as the maximal index of $x_{*} \circ \partial_{K}(E) \in H^{2}(K, T)$, where the maximum is taken over all field extensions $K / k$ and over all $E \in H^{1}(K, \bar{G})$. In fact, this maximal value is always attained in the case where $E=E_{\text {vers }} \rightarrow \operatorname{Spec}(K)$ is a versal $G$-torsor (for a suitable field $K$ ). That is,

$$
\operatorname{ind}^{x}(G, T)=\operatorname{ind}\left(x_{*} \circ \partial_{K}\left(E_{\text {vers }}\right)\right)
$$

for every $x \in X(T)$; see [Merkurjev 2013, Theorem 6.1]. Finally, we set

$$
\operatorname{ind}(G, T):=\min \left\{\sum_{i=1}^{r} \operatorname{ind}^{x_{i}}(G, T) \mid x_{1}, \ldots, x_{r} \text { generate } X(T)\right\} .
$$

Our starting point for the proof of the main theorems is the following proposition.

Proposition 2.1. Assume that the image of every $E \in H^{1}(K, \bar{G})$ under

$$
\text { д: } H^{1}(K, \bar{G}) \rightarrow H^{2}(K, T)
$$


is $p$-torsion for every field extension $K / k$. Then

(a) $\operatorname{ed}_{p}(G) \geqslant \operatorname{ind}(G, T)-\operatorname{dim}(G)$,

(b) $\operatorname{ed}(G) \leqslant \operatorname{ind}(G, T)+\operatorname{ed}(\bar{G})-r$ and $\operatorname{ed}_{p}(G) \leqslant \operatorname{ind}(G, T)+\operatorname{ed}_{p}(\bar{G})-r$.

These bounds are variants of results that have previously appeared in the literature. Part (a) is a generalization of [Brosnan et al. 2011, Corollary 4.2] (where $r$ is taken to be 1). In the case where $T$ is $\mu_{p}^{r}$, rather than $\mathbb{G}_{m}^{r}$, a variant of part (a) is proved in [Reichstein 2010, Theorem 4.1] (see also [Merkurjev 2013, Theorem 6.2]) and a variant of part (b) in [Merkurjev 2013, Corollaries 5.8 and 5.12].

Our proof of Proposition 2.1 proceeds along the same lines as these earlier proofs; it relies on the notions of essential and canonical dimension of a gerbe (for which we refer the reader to [Brosnan et al. 2011] and [Merkurjev 2013]), and the computation of the canonical dimension of a product of $p$-primary Brauer-Severi varieties in [Karpenko and Merkurjev 2008, Theorem 2.1]. In fact, the argument is easier for $T=\mathbb{G}_{m}^{r}$ than for $\mu_{p}^{r}$. In the former case (which is of interest to us here), the essential dimension of a gerbe banded by $T$ is readily expressible in terms of its canonical dimension (see formula (15) below), while an analogous formula for gerbes banded by $\mu_{p}^{r}$ requires a far greater effort to prove. (For $r=1$, compare the proofs of parts (a) and (b) of [Brosnan et al. 2011, Theorem 4.1]. For arbitrary $r \geqslant 1$, see [Karpenko and Merkurjev 2008, Theorem 3.1] or [Merkurjev 2013, Theorem 5.11].)

Proof. If $K / k$ is a field, and $E \in H^{1}(K, G)$, i.e., $E \rightarrow \operatorname{Spec}(K)$ is a $\bar{G}$-torsor, then the quotient stack $[E / G]$ is a gerbe over $\operatorname{Spec}(K)$ banded by $T$. By [Brosnan et al. 2011, Corollary 3.3] and [Merkurjev 2013, Corollary 5.7],

$\operatorname{ed}(G) \geqslant \max _{K, E} \operatorname{ed}([E / G])-\operatorname{dim}(\bar{G}) \quad$ and $\quad \operatorname{ed}_{p}(G) \geqslant \max _{K, E} \operatorname{ed}_{p}([E / G])-\operatorname{dim}(\bar{G})$,

where the maximum is taken over all field extensions $K / k$ and all $E \in H^{1}(K, \bar{G})$. On the other hand, by [Lötscher 2013, Example 3.4(i)],

$\operatorname{ed}(G) \leqslant \operatorname{ed}(\bar{G})+\max _{K, E} \operatorname{ed}([E / G]) \quad$ and $\quad \operatorname{ed}_{p}(G) \leqslant \operatorname{ed}_{p}(\bar{G})+\max _{K, E} \operatorname{ed}_{p}([E / G])$

see also [Merkurjev 2013, Corollary 5.8]. Since $\operatorname{dim}(G)=\operatorname{dim}(\bar{G})+r$, it remains to show that

$$
\max _{K, E} \operatorname{ed}([E / G])=\max _{K, E} \operatorname{ed}_{p}([E / G])=\operatorname{ind}(G, T)-r .
$$

Choose a $\mathbb{Z}$-basis $x_{1}, \ldots, x_{r}$ for the character group $X(T) \simeq \mathbb{Z}^{r}$ and let $P:=$ $P_{1} \times \cdots \times P_{r}$, where $P_{i}$ is the Brauer-Severi variety associated to $\left(x_{i}\right)_{*} \circ \partial(E) \in$ $H^{2}\left(K, \mathbb{G}_{m}\right)$. Since $T$ is a special group (i.e., every $T$-torsor over every field $K / k$ is split), the set $[E / G](K)$ of isomorphism classes of $K$-points of $[E / G]$ consists 
of exactly one element if $P(K) \neq \varnothing$ and is empty otherwise. Thus

$$
\operatorname{ed}([E / G])=\operatorname{cdim}(P) \text { and } \quad \operatorname{ed}_{p}([E / G])=\operatorname{cdim}_{p}(P),
$$

where $\operatorname{cdim}(P)$ denotes the canonical dimension of $P$. (The same argument is used in the proof of [Brosnan et al. 2011, Theorem 4.1(a)] in the case where $r=1$.) Since we are assuming that $\partial(E)$ is $p$-torsion, the index of each Brauer-Severi variety $P_{i}$ is a power of $p$. Thus by [Karpenko and Merkurjev 2008, Theorem 2.1], $\operatorname{cdim}(P)=\operatorname{cdim}_{p}(P)=\min \left\{\sum_{i=1}^{r} \operatorname{ind}\left(\left(x_{i}\right)_{*} \circ \partial_{K}(E)\right) \mid x_{1}, \ldots, x_{r}\right.$ generate $\left.X(T)\right\}-r ;$ see also [Merkurjev 2013, Theorem 4.14]. Taking $E:=E_{\text {vers }}$ to be a versal $\bar{G}$-torsor, we obtain

$$
\left.\operatorname{cdim}(P)=\operatorname{cdim}_{p}(P)=\min \left\{\sum_{i=1}^{r} \operatorname{ind}^{x_{i}}(G, T)\right) \mid x_{1}, \ldots, x_{r} \text { generate } X(T)\right\}-r ;
$$

see (12). By the definition (13) of ind $(G, T)$, the last formula can be rewritten as $\operatorname{cdim}(P)=\operatorname{cdim}_{p}(P)=\operatorname{ind}(G, T)-r$. Combining these equalities with (15), we obtain (14).

Remark 2.2. Our strategy for proving Theorem 1.1 will be to apply Proposition 2.1 to the exact sequence (11) with $G=\left(\mathrm{GL}_{p^{a_{1}}} \times \cdots \times \mathrm{GL}_{p^{a_{r}}}\right) / C$, and $T:=\mathbb{G}_{m}^{r} / C$. The only remaining issue is to find an expression for $\operatorname{ind}(G, T)$ in terms of $\operatorname{Code}(C)$.

Usually, the term ind $(G, T)$ is computed using the formula

$$
\text { ind }^{x}(G, T)=\operatorname{gcd} \operatorname{dim}(\rho),
$$

as $\rho: G \rightarrow \mathrm{GL}(V)$ ranges over all finite-dimensional representations of $G$ such that $\tau \in T$ acts on $V$ via scalar multiplication by $x(\tau)$. See, for example, [Karpenko and Merkurjev 2008, Theorem 4.4] or [Merkurjev 2013, Theorem 6.1] or [Lötscher et al. 2013, Theorem 3.1]. We will not use this approach in the present paper. Instead, we will compute the values of ind ${ }^{x}(G, T)$ and $\operatorname{ind}(G, T)$ directly from the definition, using the description of the connecting map $\partial: H^{1}(K, \bar{G}) \rightarrow H^{2}(K, T)$ given by Theorem A.1; see the proof of Proposition 4.1 below.

\section{Minimal bases}

To carry out the program outlined in Remark 2.2, we will need the notion of a minimal basis. This section will be devoted to developing this notion.

The general setting is as follows. Let $R$ be a local ring with maximal ideal $I \subset R$ and $A$ be a finitely generated $R$-module. We will refer to a generating set $S \subset A$ as a basis if no proper subset of $S$ generates $A$. In the sequel we will specialize $R$ to $\mathbb{Z} / p^{a} \mathbb{Z}$ and $A$ to a submodule of $\left(\mathbb{Z} / p^{a_{1}} \mathbb{Z}\right) \times \cdots \times\left(\mathbb{Z} / p^{a_{t}} \mathbb{Z}\right)$, where 
$a=\max \left(a_{1}, \ldots, a_{r}\right)$. However, in this section it will be convenient for us to work over an arbitrary local ring $R$.

Let $\pi: A \rightarrow A / I A$ be the natural projection. We will repeatedly appeal to Nakayama's lemma, which asserts that a subset $S \subset A$ generates $A$ as an $R$-module if and only if $\pi(S)$ generates $A / I A$ as an $R / I$-vector space; see [Lang 2002, Section X.4].

By a weight function on $A$ we shall mean any function $w: A \rightarrow \mathbb{N}$, where $\mathbb{N}$ denotes the set of nonnegative integers. We will fix $w$ throughout and will sometimes refer to w $(y)$ as the weight of $y \in A$. For each basis $B=\left\{y_{1}, \ldots, y_{t}\right\}$ of $A$, we will define the profile of $B$ as

$$
\mathrm{w}(B):=\left(\mathrm{w}\left(y_{1}\right), \ldots, \mathrm{w}\left(y_{t}\right)\right) \in \mathbb{N}^{t},
$$

where $y_{1}, \ldots, y_{t}$ are ordered so that $\mathrm{w}\left(y_{1}\right) \leqslant \mathrm{w}\left(y_{2}\right) \leqslant \cdots \leqslant \mathrm{w}\left(y_{t}\right)$. Let $\operatorname{Prof}(A) \subset \mathbb{N}^{t}$ denote the set of profiles of bases of $A$.

Proposition 3.1. $\operatorname{Prof}(A)$ has a unique minimal element with respect to the partial order on $\mathbb{N}^{t}$ given by $\left(\alpha_{1}, \ldots, \alpha_{t}\right) \preceq\left(\beta_{1}, \ldots, \beta_{t}\right)$ if $\alpha_{i} \leqslant \beta_{i}$ for every $i=1, \ldots, t$.

Note that since every descending chain in $(\operatorname{Prof}(A), \preceq)$ terminates, the unique minimal element is comparable to every element of $\operatorname{Prof}(A)$.

Proof. We argue by contradiction. Set $t:=\operatorname{dim}(A / I A)$. Suppose $X=\left\{x_{1}, \ldots, x_{t}\right\}$ and $Y=\left\{y_{1}, \ldots, y_{t}\right\}$ are bases of $A$ such that $\mathrm{w}(X)$ and $\mathrm{w}(Y)$ are distinct minimal elements of $\operatorname{Prof}(A)$. Let us order $X$ and $Y$ so that $\mathrm{w}\left(x_{1}\right) \leqslant \cdots \leqslant \mathrm{w}\left(x_{t}\right)$ and $\mathrm{w}\left(y_{1}\right) \leqslant \cdots \leqslant \mathrm{w}\left(y_{t}\right)$. Since $\mathrm{w}(X) \neq \mathrm{w}(Y)$, there exists an $s$ between 0 and $t-1$ such that

$$
\mathrm{w}\left(x_{i}\right)=\mathrm{w}\left(y_{i}\right) \text { for all } i=1, \ldots, s,
$$

but $\mathrm{w}\left(x_{s+1}\right) \neq \mathrm{w}\left(y_{s+1}\right)$. After possibly interchanging $X$ and $Y$, we may assume without loss of generality that $\mathrm{w}\left(x_{s+1}\right)<\mathrm{w}\left(y_{s+1}\right)$.

Let $\pi: A \rightarrow A / I A$ be the natural projection, as above. By Nakayama's lemma, $\pi\left(x_{1}\right), \ldots, \pi\left(x_{s+1}\right)$ are $R / I$-linearly independent in $A / I A$. Choose $t-s-1$ elements of $Y$, say $y_{j_{s+2}}, \ldots, y_{j_{t}}$, such that $\pi\left(x_{1}\right), \ldots, \pi\left(x_{s+1}\right), \pi\left(y_{j_{s+2}}\right), \ldots, \pi\left(y_{j_{t}}\right)$ form an $R / I$-basis of $A / I A$. After permuting $y_{j_{s+2}}, \ldots, y_{j_{t}}$, we may assume that $\mathrm{w}\left(y_{j_{s+2}}\right) \leqslant \cdots \leqslant \mathrm{w}\left(y_{j_{t}}\right)$. Applying Nakayama's lemma once again, we see that $Z=\left\{x_{1}, \ldots, x_{s+1}, y_{j_{s+2}}, \ldots, y_{j_{t}}\right\}$ is a basis of $A$.

We claim that $\mathrm{w}(Z) \prec \mathrm{w}(Y)$, where the inequality is strict. Since we assumed that $\mathrm{w}(Y)$ is minimal in $\operatorname{Prof}(A)$, this claim leads to a contradiction, thus completing the proof of Proposition 3.1.

To prove the claim, let $z_{1}, \ldots, z_{t}$ be the elements of $Z$, in increasing order of their weight: $\mathrm{w}\left(z_{1}\right) \leqslant \mathrm{w}\left(z_{2}\right) \leqslant \cdots \leqslant \mathrm{w}\left(z_{t}\right)$. It suffices to show that $\mathrm{w}\left(z_{i}\right) \leqslant \mathrm{w}\left(y_{i}\right)$ for every $i=1, \ldots, t$, and $\mathrm{w}\left(z_{s+1}\right)<\mathrm{w}\left(y_{s+1}\right)$. Let us consider three cases. 
Case 1: $i \leqslant s$. Since

$$
\mathrm{w}\left(x_{1}\right)=\mathrm{w}\left(y_{1}\right) \leqslant \mathrm{w}\left(x_{2}\right)=\mathrm{w}\left(y_{2}\right) \leqslant \cdots \leqslant \mathrm{w}\left(x_{i}\right)=\mathrm{w}\left(y_{i}\right),
$$

$Z$ has at least $i$ elements whose weight is at most $\mathrm{w}\left(y_{i}\right)$, namely $x_{1}, \ldots, x_{i}$. Thus $\mathrm{w}\left(z_{i}\right) \leqslant \mathrm{w}\left(y_{i}\right)$.

Case 2: $i=s+1$. $Z$ has at least $s+1$ elements, namely $x_{1}, \ldots, x_{s+1}$ whose weight is at most $\mathrm{w}\left(x_{s+1}\right)$. Hence, $\mathrm{w}\left(z_{s+1}\right) \leqslant \mathrm{w}\left(x_{s+1}\right)<\mathrm{w}\left(y_{s+1}\right)$, as desired.

Case 3: $i>s+1$. Recall that both $y_{1}, \ldots, y_{t}$ and $y_{j_{s+2}}, \ldots, y_{j_{t}}$ are arranged in weight-increasing order. For any $i \geqslant s+2$, there are at least $t-i+1$ elements of $Y$ whose weight is at least $\mathrm{w}\left(y_{j_{i}}\right)$, namely $y_{j_{i}}, y_{j_{i+1}}, \ldots, y_{j_{t}}$. Thus

$$
\mathrm{w}\left(y_{j_{i}}\right) \leqslant \mathrm{w}\left(y_{i}\right)
$$

for any $i=s+2, \ldots, t$. Consequently, $Z$ has at least $i$ elements of weight at most $\mathrm{w}\left(y_{i}\right)$, namely $x_{1}, \ldots, x_{s+1}, y_{j_{s+2}}, \ldots, y_{j_{i}}$. Hence, $\mathrm{w}\left(z_{i}\right) \leqslant \mathrm{w}\left(y_{i}\right)$, as desired.

This completes the proof of the claim and hence of Proposition 3.1.

Definition 3.2. We will say that a basis $y_{1}, \ldots, y_{t}$ of $A$ is minimal if its profile is the minimal element of $\operatorname{Prof}(A)$, as in Proposition 3.1. Note that a minimal basis in $A$ is usually not unique; however, any two minimal bases have the same profile in $\mathbb{N}^{t}$.

Remark 3.3. We can construct a minimal basis of $A$ using the following "greedy algorithm". Select $y_{1} \in A$ of minimal weight, subject to the condition that $\pi\left(y_{1}\right) \neq 0$. Next select $y_{2}$ of minimal weight, subject to the condition that $\pi\left(y_{1}\right)$ and $\pi\left(y_{2}\right)$ are $R / I$-linear independent in $A / I A$. Then select $y_{3}$ of minimal weight, subject to the condition that $\pi\left(y_{1}\right), \pi\left(y_{2}\right)$ and $\pi\left(y_{3}\right)$ are $R / I$-linear independent in $A / I A$. Continue recursively. After $t=\operatorname{dim}_{R / I}(A / I A)$ steps, we obtain a minimal basis $y_{1}, \ldots, y_{t}$ for $A$.

Example 3.4. Set $R:=\mathbb{F}_{p}, I:=(0), G$ a finite $p$-group, $D:=Z(G)[p]$ the subgroup of $p$-torsion elements of the center $Z(G)$, and $A:=X(D)$ the group of characters of $D$. For $x \in A$, define $\mathrm{w}(x)$ to be the minimal dimension of a representation $G \rightarrow \operatorname{GL}\left(V_{x}\right)$, such that $D$ acts on $V_{x}$ via scalar multiplication by $x$. If $\left\{x_{1}, \ldots, x_{t}\right\}$ is a minimal basis of $A$, then $V_{x_{1}} \oplus \cdots \oplus V_{x_{t}}$ is a faithful representation of $G$ of minimal dimension; see [Karpenko and Merkurjev 2008, Remark 4.7].

\section{Conclusion of the proof of Theorem 1.1}

Recall that we are interested in the essential dimension of the group

$$
G=\left(\mathrm{GL}_{p^{a_{1}}} \times \cdots \times \mathrm{GL}_{p^{a_{r}}}\right) / C
$$

where $C$ is a subgroup of $\mu:=\mu_{p^{a_{1}}} \times \cdots \times \mu_{p^{a_{r}}}$. We will think of the group of characters $X\left(\mathbb{G}_{m}^{r}\right)$ as $\mathbb{Z}^{r}$ by identifying the character $x\left(\tau_{1}, \ldots, \tau_{r}\right)=\tau_{1}^{m_{1}} \cdots \tau_{r}^{m_{r}}$ 
with $\left(m_{1}, \ldots, m_{r}\right) \in \mathbb{Z}^{r}$. Characters of $T:=\mathbb{G}_{m}^{r} / C$ are identified in this manner with the $r$-tuples $\left(m_{1}, \ldots, m_{r}\right) \in \mathbb{Z}^{r}$ such that $\tau_{1}^{m_{1}} \cdots \tau_{r}^{m_{r}}=1$ for every $\left(\tau_{1}, \ldots, \tau_{r}\right) \in C$. The relationship among these character groups is illustrated by the following diagram:

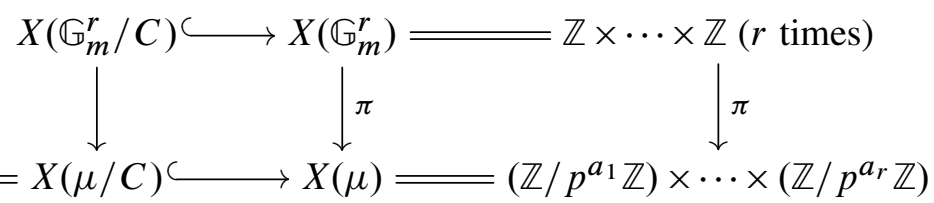

Here $\operatorname{Code}(C)$ is as in (8) and $\pi$ is the natural projection, given by restricting a character from $\mathbb{G}_{m}^{r}$ to $\mu$.

Our proof of Theorem 1.1 will be based on the strategy outlined in Remark 2.2. In view of Proposition 2.1 it suffices to establish the following:

Proposition 4.1. Consider the central exact sequence

$$
1 \rightarrow T \rightarrow G \rightarrow \bar{G} \rightarrow 1,
$$

where $G=\left(\mathrm{GL}_{p^{a_{1}}} \times \cdots \times \mathrm{GL}_{p^{a_{r}}}\right) / C, C$ is a subgroup of $\mu:=\mu_{p^{a_{1}}} \times \cdots \times \mu_{p^{a_{r}}}$, $T:=\mathbb{G}_{m}^{r} / C$ and $\bar{G}:=\mathrm{PGL}_{p^{a_{1}}} \times \cdots \times \mathrm{PGL}_{p}{ }^{a_{r}}$.

(a) If $x \in X(T)$ and $y=\pi(x) \in \operatorname{Code}(C)$ then ind $^{x}(G, T)=p^{\mathrm{w}(y)}$.

(b) $\operatorname{ind}(G, T)=p^{\mathrm{w}\left(z_{1}\right)}+\cdots+p^{\mathrm{w}\left(z_{t}\right)}+r-t$, where $z_{1}, \ldots, z_{t}$ is a minimal basis of $\operatorname{Code}(C)$.

Proof of Proposition 4.1(a). Consider the connecting map $\partial: H^{1}(K, \bar{G}) \rightarrow H^{2}(K, T)$ associated to the central exact sequence (16). Given a character $x: T \rightarrow \mathbb{G}_{m}$, $x\left(\tau_{1}, \ldots, \tau_{r}\right)=\tau_{1}^{m_{1}} \cdots \tau_{r}^{m_{r}}$, ind ${ }^{x}(G, T)$ is, by definition, the maximal value of $\operatorname{ind}\left(x_{*} \partial(E)\right)$, as $K$ ranges over all fields containing $k$ and $E$ ranges over $H^{1}(K, \bar{G})$. In this case, $\bar{G}=\mathrm{PGL}_{p^{a_{1}}} \times \cdots \times \mathrm{PGL}_{p^{a_{r}}}$, and thus $H^{1}(K, \bar{G})$ is the set of $r$ tuples $\left(A_{1}, \ldots, A_{r}\right)$ of central simple algebras, where the degree of $A_{i}$ is $p^{a_{i}}$. The group $H^{2}\left(K, \mathbb{G}_{m}\right)$ is naturally identified with the Brauer group $\operatorname{Br}(K)$, and the map $x_{*} \partial$ takes an $r$-tuple $\left(A_{1}, \ldots, A_{r}\right)$, as above, to the Brauer class of $A:=A_{1}^{\otimes m_{1}} \otimes \cdots \otimes A_{r}^{\otimes m_{r}}$.

Since $\operatorname{deg}\left(A_{i}\right)=p^{a_{i}}$, the Brauer class of $A$ depends only on

$$
y=\pi(x)=\left(m_{1} \bmod p^{a_{1}}, \ldots, m_{r} \bmod p^{a_{r}}\right) \in\left(\mathbb{Z} / p^{a_{1}} \mathbb{Z}\right) \times \cdots \times\left(\mathbb{Z} / p^{a_{r}} \mathbb{Z}\right) .
$$

Moreover, if $m_{i} \equiv u_{i} p^{e_{i}}\left(\bmod p^{a_{i}}\right)$, where $u_{i}$ is prime to $p$ and $0 \leqslant e_{i} \leqslant a_{i}$, then $\operatorname{ind}\left(A_{i}^{\otimes m_{i}}\right) \leqslant p^{a_{i}-e_{i}}$. Now recall that $\mathrm{w}(y)$ is defined as $\sum_{i=1}^{r}\left(a_{i}-e_{i}\right)$. Thus

$$
\operatorname{ind}(A) \leqslant \prod_{i=1}^{r} \operatorname{ind}\left(A_{i}^{\otimes m_{i}}\right) \leqslant \prod_{i=1}^{r} p^{a_{i}-e_{i}}=p^{\mathrm{w}(y)} .
$$


To prove the opposite inequality, we set $A_{i}$ to be the symbol algebra $\left(\alpha_{i}, \beta_{i}\right)_{p^{a_{i}}}$, over the field $K=k(\zeta)\left(\alpha_{1}, \ldots, \alpha_{r}, \beta_{1}, \ldots, \beta_{r}\right)$, where $\zeta$ is a primitive root of unity of degree $p^{\max \left(a_{1}, \ldots, a_{r}\right)}$ and $\alpha_{1}, \ldots, \alpha_{r}, \beta_{1}, \ldots, \beta_{r}$ are $2 r$ independent variables over $k$. Writing $m_{i}=u_{i} p^{e_{i}}$, as above, we see that $A_{i}^{\otimes m_{i}}$ is Brauer equivalent to $B_{i}:=\left(\alpha_{i}, \beta_{i}^{u_{i}}\right)_{p^{a_{i}-e_{i}}}$ over $K$. An easy valuation-theoretic argument shows that $B:=B_{1} \otimes_{K} \cdots \otimes_{K} B_{t}$ is a division algebra. (In particular, the norm form of $B$ is a Pfister polynomial and hence, is anisotropic; see [Reichstein 1999, Theorem 3.2 and Proposition 3.4].) Thus

$$
\operatorname{ind}(A)=\operatorname{ind}(B)=\operatorname{ind}\left(B_{1}\right) \cdots \cdot \operatorname{ind}\left(B_{t}\right)=p^{\left(a_{1}-e_{1}\right)+\cdots+\left(a_{t}-e_{t}\right)}=p^{\mathrm{w}(y)},
$$

as desired. We conclude that ind ${ }^{x}(G, T) \geqslant \operatorname{ind}(A)=p^{\mathrm{w}(y)}$, thus completing the proof of Proposition 4.1(a).

Our proof of Proposition 4.1(b) will rely on the following elementary lemma.

Lemma 4.2. Let $p$ be a prime, $M$ be a finite abelian $p$-group, and $f: \mathbb{Z}^{n} \rightarrow M$ be a surjective $\mathbb{Z}$-module homomorphism for some $n \geqslant 1$. Then for every basis $y_{1}, \ldots, y_{t}$ of $M$, there exists $a \mathbb{Z}$-basis $x_{1}, \ldots, x_{n}$ of $\mathbb{Z}^{n}$ and an integer $c$ prime to $p$ such that $f\left(x_{1}\right)=c y_{1}, f\left(x_{2}\right)=y_{2}, \ldots, f\left(x_{t}\right)=y_{t}$ and $f\left(x_{t+1}\right)=\cdots=f\left(x_{n}\right)=0$.

Proof. By [Lang 2002, Theorem III.7.8], there exists a basis $e_{1}, \ldots, e_{n}$ of $\mathbb{Z}^{n}$ such that $\operatorname{Ker}(f)$ is generated by $p^{d_{i}} e_{i}$ for some integers $d_{1}, \ldots, d_{t} \geqslant 0$. Since $M$ has rank $t$, we may assume without loss of generality that $d_{1}, \ldots, d_{t} \geqslant 1$ and $d_{t+1}=\cdots=d_{n}=0$. That is, we may identify $M$ with $\left(\mathbb{Z} / p^{d_{1}} \mathbb{Z}\right) \times \cdots \times\left(\mathbb{Z} / p^{d_{t}} \mathbb{Z}\right)$ and assume that

$$
f\left(r_{1}, \ldots, r_{n}\right)=\left(r_{1} \bmod p^{d_{1}}, \ldots, r_{t} \bmod p^{d_{t}}\right) \quad \forall\left(r_{1}, \ldots, r_{n}\right) \in \mathbb{Z}^{n} .
$$

It now suffices to lift $c y_{1}, \ldots, y_{t} \in M$ to a basis $x_{1}, \ldots, x_{t}$ of $\mathbb{Z}^{t}$ for a suitable integer $c$, prime to $p$. Indeed, if we manage to do this, then we will obtain a basis of $\mathbb{Z}^{n}$ of the desired form by appending

$$
x_{t+1}:=e_{t+1}, \ldots, x_{n}:=e_{n} \in \operatorname{Ker}(f)
$$

to $x_{1}, \ldots, x_{t}$. Thus we may assume that $n=t$.

Now observe that $f: \mathbb{Z}^{n} \rightarrow M$, factors as $\mathbb{Z}^{n} \rightarrow\left(\mathbb{Z} / p^{d} \mathbb{Z}\right)^{n} \rightarrow M$, where $d:=$ $\max \left(d_{1}, \ldots, d_{t}\right)$. Lift each $y_{i} \in M$ to some $y_{i}^{\prime} \in\left(\mathbb{Z} / p^{d} \mathbb{Z}\right)^{n}$. By Nakayama's lemma, $y_{1}^{\prime}, \ldots, y_{n}^{\prime}$ form a $\mathbb{Z} / p^{d} \mathbb{Z}$-basis of $\left(\mathbb{Z} / p^{d} \mathbb{Z}\right)^{n}$. It now suffices to lift $c y_{1}^{\prime}, y_{2}^{\prime}, \ldots, y_{n}^{\prime}$ to a basis of $\mathbb{Z}^{n}$ for a suitable integer $c$, prime to $p$. In other words, we may assume without loss of generality that $M=\left(\mathbb{Z} / p^{d} \mathbb{Z}\right)^{n}$, and $f: \mathbb{Z}^{n} \rightarrow\left(\mathbb{Z} / p^{d} \mathbb{Z}\right)^{n}$ is the natural projection.

Now suppose $y_{i}=\left(y_{i 1}, \ldots, y_{i n}\right)$ for some $y_{i j} \in \mathbb{Z} / p^{d} \mathbb{Z}$. Since $y_{1}, \ldots, y_{m}$ form a basis of $\left(\mathbb{Z} / p^{d} \mathbb{Z}\right)^{n}$, the matrix $A=\left(y_{i j}\right)$ is invertible, i.e., $A \in \mathrm{GL}_{n}\left(\mathbb{Z} / p^{d} \mathbb{Z}\right)$. 
After rescaling $y_{1}$ by $c:=\operatorname{det}(A)^{-1}$ in $\mathbb{Z} / p^{d} \mathbb{Z}$, we may assume that $\operatorname{det}(A)=1$. The lemma now follows from the surjectivity of the natural projection

$$
\mathrm{SL}_{t}(\mathbb{Z}) \rightarrow \mathrm{SL}_{t}\left(\mathbb{Z} / p^{d} \mathbb{Z}\right)
$$

see [Shimura 1971, Lemma 1.38].

Proof of Proposition 4.1(b). By definition, ind $(G, T)$ is the minimal value of ind $^{x_{1}}(G, T)+\cdots+$ ind $^{x_{r}}(G, T)$, as $x_{1}, \ldots, x_{r}$ range over the bases of $X(T) \subset \mathbb{Z}^{r}$. By part (a), we can rewrite this as

$$
\operatorname{ind}(G, T)=\min \left\{p^{\mathrm{w}\left(\pi\left(x_{1}\right)\right)}+\cdots+p^{\mathrm{w}\left(\pi\left(x_{r}\right)\right)} \mid x_{1}, \ldots, x_{r} \text { is a } \mathbb{Z} \text {-basis of } X(T)\right\} .
$$

Here, as before, $\pi\left(x_{i}\right) \in \operatorname{Code}(C)$ is the restriction of $x_{i}$ from $T=\mathbb{G}_{m}^{r} / C$ to $\mu / C$.

Let $z_{1}, \ldots, z_{t} \in \operatorname{Code}(C)$ be a minimal basis, as in the statement of the proposition. We will prove part (b) by showing that

(i) $p^{\mathrm{w}\left(\pi\left(x_{1}\right)\right)}+\cdots+p^{\mathrm{w}\left(\pi\left(x_{r}\right)\right)} \geqslant p^{\mathrm{w}\left(z_{1}\right)}+\cdots+p^{\mathrm{w}\left(z_{t}\right)}+r-t$ for every $\mathbb{Z}$-basis $x_{1}, \ldots, x_{r}$ of $X(T)$, and

(ii) there exists a particular $\mathbb{Z}$-basis $x_{1}, \ldots, x_{r}$ of $X(T)$ such that $p^{\mathrm{w}\left(\pi\left(x_{1}\right)\right)}+\cdots+$ $p^{\mathrm{w}\left(\pi\left(x_{r}\right)\right)}=p^{\mathrm{w}\left(z_{1}\right)}+\cdots+p^{\mathrm{w}\left(z_{t}\right)}+r-t$.

To prove (i), note that if $x_{1}, \ldots, x_{r}$ form a $\mathbb{Z}$-basis of $X(T)$, then $\pi\left(x_{1}\right), \ldots, \pi\left(x_{r}\right)$ form a generating set for $\operatorname{Code}(C)$. By Nakayama's lemma, every generating set for $\operatorname{Code}(C)$ contains a basis. After renumbering $x_{1}, \ldots, x_{r}$, we may assume that $\pi\left(x_{1}\right), \ldots, \pi\left(x_{t}\right)$ is a basis of $\operatorname{Code}(C)$ and $\mathrm{w}\left(\pi\left(x_{1}\right)\right) \leqslant \cdots \leqslant \mathrm{w}\left(\pi\left(x_{t}\right)\right)$. By Proposition $3.1, \mathrm{w}\left(z_{i}\right) \leqslant \mathrm{w}\left(\pi\left(x_{i}\right)\right)$ for every $i=1, \ldots, t$. Thus

$$
\begin{aligned}
p^{\mathrm{w}\left(\pi\left(x_{1}\right)\right)}+\cdots+p^{\mathrm{w}\left(\pi\left(x_{r}\right)\right)} & \\
& \geqslant p^{\mathrm{w}\left(\pi\left(x_{1}\right)\right)}+\cdots+p^{\mathrm{w}\left(\pi\left(x_{t}\right)\right)}+\underbrace{p^{0}+\cdots+p^{0}}_{r-t \text { times }} \geqslant p^{\mathrm{w}\left(z_{1}\right)}+\cdots+p^{\mathrm{w}\left(z_{t}\right)}+r-t .
\end{aligned}
$$

To prove (ii), recall that by Lemma 4.2 there exists an integer $c$, prime to $p$, and a $\mathbb{Z}$-basis $x_{1}, \ldots, x_{r}$ of $X(T)$ such that $\pi\left(x_{1}\right)=c z_{1}, \pi\left(x_{2}\right)=z_{2}, \ldots, \pi\left(x_{t}\right)=z_{t}$, and $\pi\left(x_{t+1}\right)=\cdots=\pi\left(x_{r}\right)=0$. Since $c$ is prime to $p, \mathrm{w}\left(c z_{1}\right)=\mathrm{w}\left(z_{1}\right)$. Thus for this particular choice of $x_{1}, \ldots, x_{r}$, we have

$$
\begin{aligned}
& p^{\mathrm{w}\left(\pi\left(x_{1}\right)\right)}+\cdots+p^{\mathrm{w}\left(\pi\left(x_{r}\right)\right)}= \\
& p^{\mathrm{w}\left(c z_{1}\right)}+p^{\mathrm{w}\left(z_{2}\right)}+\cdots+p^{\mathrm{w}\left(z_{t}\right)}+\underbrace{p^{0}+\cdots+p^{0}}_{r-t \text { times }}=p^{\mathrm{w}\left(z_{1}\right)}+\cdots+p^{\mathrm{w}\left(z_{t}\right)}+r-t,
\end{aligned}
$$

as desired. 


\section{Proof of Theorem 1.2}

Consider the action of a linear algebraic group $\Gamma$ on an absolutely irreducible algebraic variety $X$ defined over $k$. We say that a subgroup $S \subset \Gamma$ is a stabilizer in general position for this action if there exists a dense open subset $U \subset X$ such that the scheme-theoretic stabilizer $\operatorname{Stab}_{\Gamma}(x)$ is conjugate to $S$ over $\bar{k}$ for every $x \in U(\bar{k})$. Here, as usual, $\bar{k}$ denotes the algebraic closure of $k$. In the sequel we will not specify $U$ and will simply say that $\operatorname{Stab}_{\Gamma}(x)$ is conjugate to $S$ for $x \in X(\bar{k})$ in general position. Note that a stabilizer in general position $S$ for a $\Gamma$-action on $X$ does not always exist, and when it does, it is usually not unique. However, over $\bar{k}$, $S$ is unique up to conjugacy.

For the rest of this section we will always assume that $\operatorname{char}(k)=0$. A theorem of R. W. Richardson [1972] tells us that under this assumption every linear action of a reductive group $\Gamma$ on a vector space $V$ has a stabilizer $S \subset \Gamma$ in general position. Note that in [Richardson 1972], $k$ is assumed to be algebraically closed. Thus a priori the subgroup $S$ and the open subset $U \subset V$, where all stabilizers are conjugate to $S$, are only defined over $\bar{k}$. However, $U$ has only finitely many Galois translates. After replacing $U$ by the intersection of all of these translates, we may assume that $U$ is defined over $k$. Moreover, we may take $S:=\operatorname{Stab}_{G}(x)$ for some $k$-point $x \in U(k)$ and thus assume that $S$ is defined over $k$. For a detailed discussion of stabilizers in general position over an algebraically closed field of characteristic zero, see [Popov and Vinberg 1994, Section 7].

We will say that a $\Gamma$-action on $X$ is generically free if the trivial subgroup $S=\left\{1_{\Gamma}\right\} \subset \Gamma$ is a stabilizer in general position for this action.

Lemma 5.1. Let $\Gamma$ be a reductive linear algebraic group and $\rho: \Gamma \rightarrow \mathrm{GL}(V)$ be a finite-dimensional representation. If $\operatorname{Stab}_{\Gamma}(v)$ is central in $\Gamma$ for $v \in V$ in general position, then the induced action of $\Gamma / \operatorname{Ker}(\rho)$ on $V$ is generically free.

Proof. Let $S \subset \Gamma$ be the stabilizer in general position for the $\Gamma$-action on $V$. Clearly $\operatorname{Ker}(\rho) \subset S$. We claim that, in fact, $\operatorname{Ker}(\rho)=S$; the lemma easily follows from this claim.

To prove the opposite inclusion, $S \subset \operatorname{Ker}(\rho)$, note that under the assumption of the lemma, $S$ is central in $\Gamma$. Let $U \subset V$ be a dense open subset such that the stabilizer of every $v \in U(\bar{k})$ is conjugate to $S$. Since $S$ is central, $\operatorname{Stab}_{\Gamma}(v)$ is, in fact, equal to $S$. In other words, $S$ stabilizes every point in $U$ and thus every point in $V$. That is, $S \subset \operatorname{Ker}(\rho)$, as claimed.

Our interest in generically free actions in this section has to do with the following fact: if there exists a generically free linear representation $G \rightarrow \mathrm{GL}(V)$ then

$$
\operatorname{ed}(G) \leqslant \operatorname{dim}(V)-\operatorname{dim}(G)
$$


see, e.g., [Reichstein 2010, (2.3)] or [Merkurjev 2013, Proposition 3.13]. This inequality will play a key role in our proof of Theorem 1.2.

Now set $\Gamma:=\mathrm{GL}_{n_{1}} \times \cdots \times \mathrm{GL}_{n_{r}}$ and $\Gamma^{\prime}:=\mathrm{SL}_{n_{1}} \times \cdots \times \mathrm{SL}_{n_{r}}$. Let $V_{i}$ be the natural $n_{i}$-dimensional representation, $V_{i}^{-1}$ be the dual representation, and $V_{i}^{0}$ be the trivial 1-dimensional representation of $\mathrm{GL}_{n_{i}}$. For $\epsilon=\left(\epsilon_{1}, \ldots, \epsilon_{r}\right)$, where each $\epsilon_{i}$ is $-1,0$ or 1 , we define $\rho_{\epsilon}$ to be the natural representation of $\Gamma$ on the tensor product

$$
V_{\epsilon}=V_{1}^{\epsilon_{1}} \otimes \cdots \otimes V_{r}^{\epsilon_{r}} .
$$

Lemma 5.2. Suppose $2 \leqslant n_{1} \leqslant \cdots \leqslant n_{r} \leqslant \frac{1}{2} n_{1} \cdots n_{r-1}$, and

$$
\left(n_{1}, \ldots, n_{r}\right) \neq(2,2,2,2),(3,3,3) \text { or }(2, n, n) \quad \text { for any } n \geqslant 2 \text {. }
$$

If $\epsilon=\left(\epsilon_{1}, \ldots, \epsilon_{r}\right) \in\{ \pm 1\}^{r}$, then the induced action of $\Gamma / \operatorname{Ker}\left(\rho_{\epsilon}\right)$ on $V_{\epsilon}$ is generically free.

Proof. By Lemma 5.1 it suffices to prove the following claim: the stabilizer $\operatorname{Stab}_{\Gamma}(v)$ is central in $\Gamma$ for $v \in V_{\epsilon}$ in general position. To prove this claim, we may assume without loss of generality that $k$ is algebraically closed.

We first reduce to the case where $\epsilon=(1, \ldots, 1)$. Suppose the claim is true in this case, and let $\left(\epsilon_{1}, \ldots, \epsilon_{r}\right) \in\{ \pm 1\}^{r}$. By choosing bases of $V_{1}, \ldots, V_{r}$, we can identify $V_{i}$ with $V_{i}^{\epsilon_{i}}$ (we can take the identity map if $\epsilon_{i}=1$ ). Define an automorphism

$$
\begin{aligned}
\sigma: \Gamma & \rightarrow \Gamma, \\
\left(g_{1}, \ldots, g_{r}\right) & \mapsto\left(g_{1}^{*}, \ldots, g_{r}^{*}\right),
\end{aligned}
$$

where

$$
g_{i}^{*}= \begin{cases}g_{i} & \text { if } \epsilon_{i}=1, \\ \left(g_{i}^{-1}\right)^{T} & \text { if } \epsilon_{i}=-1 .\end{cases}
$$

Now $\rho_{\left(\epsilon_{1}, \ldots, \epsilon_{r}\right)}$ is isomorphic to the representation $\rho_{(1, \ldots, 1)} \circ \sigma$. Since the center of $\Gamma$ is invariant under $\sigma$, we see that the claim holds for $\rho_{\epsilon}$ as well.

From now on we will assume $\epsilon=(1, \ldots, 1)$. By [Popov 1987, Theorem 2],

$$
\Gamma / Z(\Gamma)=\mathrm{PGL}_{n_{1}} \times \cdots \times \mathrm{PGL}_{n_{r}}=\Gamma^{\prime} / Z\left(\Gamma^{\prime}\right)
$$

acts generically freely on the projective space $\mathbb{P}\left(V_{\epsilon}\right)=V_{\epsilon} / Z(\Gamma)$. In other words, for $v \in V_{\epsilon}$ in general position, the stabilizer in $\Gamma$ of the associated projective point $[v] \in \mathbb{P}\left(V_{\epsilon}\right)$ is trivial. Hence, the stabilizer of $v$ is contained in $Z(\Gamma)$; see the exact sequence in [Reichstein and Vonessen 2007, Lemma 3.1]. This completes the proof of the claim and thus of Lemma 5.2.

We are now ready to proceed with the proof of Theorem 1.2. We begin by specializing $n_{i}$ to $p^{a_{i}}$ for every $i=1, \ldots, r$, so that $\Gamma$ becomes $\mathrm{GL}_{p^{a_{1}}} \times \cdots \times \mathrm{GL}_{p^{a_{r}}}$. Let

$$
y_{1}, \ldots, y_{t} \in\left(\mathbb{Z} / p^{a_{1}} \mathbb{Z}\right) \times \cdots \times\left(\mathbb{Z} / p^{a_{r}} \mathbb{Z}\right)
$$


be a basis of $\operatorname{Code}(C)$ satisfying the conditions of Theorem 1.2. Lift each $y_{i}=$ $\left(y_{i 1}, \ldots, y_{i r}\right)$ to $x_{i}:=\left(x_{i 1}, \ldots, x_{i r}\right) \in \mathbb{Z}^{r}$ by setting $x_{i j}:=-1,0$ or 1 , depending on whether $y_{i j}$ is $-1,0$ or 1 in $\mathbb{Z} / p^{a_{j}} \mathbb{Z}$. (If $p^{a_{j}}=2$, then we define each $x_{i j}$ to be 0 or 1.) By Nakayama's lemma, the images of $y_{1}, \ldots, y_{t}$ are $\mathbb{F}_{p}$-linearly independent in $\operatorname{Code}(C) / p \operatorname{Code}(C)$. Thus the integer vectors $x_{1}, \ldots, x_{t}$ are $\mathbb{Z}$-linearly independent. (Note that, unlike in the situation of Lemma 4.2, here it will not matter to us whether $x_{1}, \ldots, x_{t}$ can be completed to a $\mathbb{Z}$-basis of $\mathbb{Z}^{r}$.) We view each $x_{i}$ as a character $\mathbb{G}_{m}^{r} \rightarrow \mathbb{G}_{m}$ and set

$$
\widetilde{C}:=\operatorname{Ker}\left(x_{1}\right) \cap \cdots \cap \operatorname{Ker}\left(x_{t}\right) \subset \mathbb{G}_{m}^{r} .
$$

Since $x_{1}, \ldots, x_{t}$ are linearly independent,

$$
\operatorname{dim}(\widetilde{C})=r-t .
$$

Set $G:=\Gamma / C$ and $\widetilde{G}:=\Gamma / \widetilde{C}$. By our construction, $\widetilde{C} \cap \mu=C$. Corollary A.2 now tells us that $\operatorname{ed}_{p}(G) \leqslant \operatorname{ed}(G)=\operatorname{ed}(\widetilde{G})$. By Theorem 1.1(a),

$$
\operatorname{ed}(G) \geqslant \operatorname{ed}_{p}(G) \geqslant\left(\sum_{i=1}^{t} p^{\mathrm{w}\left(y_{i}\right)}\right)-p^{2 a_{1}}-\cdots-p^{2 a_{r}}+r-t .
$$

It thus suffices to show that $\operatorname{ed}(\widetilde{G}) \leqslant\left(\sum_{i=1}^{t} p^{\mathrm{w}\left(y_{i}\right)}\right)-p^{2 a_{1}}-\cdots-p^{2 a_{r}}+r-t$, or equivalently,

$$
\operatorname{ed}(\widetilde{G}) \leqslant\left(\sum_{i=1}^{t} p^{\mathrm{w}\left(y_{i}\right)}\right)-\operatorname{dim}(\widetilde{G})
$$

see (19). By (17), in order to prove the last inequality, it is enough to construct a generically free linear representation of $\widetilde{G}$ of dimension $\sum_{i=1}^{t} p^{\mathrm{w}\left(y_{i}\right)}$. Such a representation is furnished by the lemma below.

Recall that $x_{i}=\left(x_{i 1}, \ldots, x_{i r}\right) \in \mathbb{Z}^{r}$, where each $x_{i j}=-1,0$ or 1 , and $\rho_{x_{i}}$ is the natural representation of $\Gamma:=\mathrm{GL}_{p^{a}} \times \cdots \times \mathrm{GL}_{p^{a r}}$ on $V_{x_{i}}:=V_{1}^{x_{i 1}} \otimes \cdots \otimes V_{r}^{x_{i r}}$, as in (18), with $\operatorname{dim}\left(V_{i}\right)=n_{i}=p^{a_{i}}$.

Lemma 5.3. Let $V=V_{x_{1}} \oplus \cdots \oplus V_{x_{t}}$ and $\rho:=\rho_{x_{1}} \oplus \cdots \oplus \rho_{x_{t}}: \Gamma \rightarrow \operatorname{GL}(V)$. Then

(a) $\operatorname{dim}(V)=p^{\mathrm{w}\left(y_{1}\right)}+\cdots+p^{\mathrm{w}\left(y_{t}\right)}$,

(b) $\operatorname{Ker}(\rho)=\widetilde{C}$, and

(c) the induced action of $\widetilde{G}=\Gamma / \widetilde{C}$ on $V$ is generically free.

Proof. For each $i=1, \ldots t$, we have

$$
\operatorname{dim}\left(V_{x_{i}}\right)=\prod_{x_{i j} \neq 0} p^{a_{j}}=\prod_{y_{i j} \neq 0} p^{a_{j}}=p^{\sum_{y_{i j} \neq 0} a_{j}} .
$$

Since each $y_{i j}=-1,0$ or $1, \sum_{y_{i j} \neq 0} a_{j}=\mathrm{w}\left(y_{i}\right)$. Thus $\operatorname{dim}\left(V_{x_{i}}\right)=p^{\mathrm{w}\left(y_{i}\right)}$, and part (a) follows. 
Now choose $v_{i} \in V_{x_{i}}$ in general position and set $v:=\left(v_{1}, \ldots, v_{r}\right)$. We claim that $\operatorname{Stab}_{\Gamma}(v)$ is central in $\Gamma$.

Suppose for a moment that this claim is established. Since the center $Z(\Gamma)=\mathbb{G}_{m}^{r}$ acts on $V_{x_{i}}$ via scalar multiplication by the character $x_{i}: \mathbb{G}_{m}^{r} \rightarrow \mathbb{G}_{m}$, we see that

$$
\operatorname{Ker}(\rho)=\operatorname{Ker}\left(\rho_{\mid \mathbb{G}_{m}^{r}}\right)=\operatorname{Ker}\left(x_{1}\right) \cap \cdots \cap \operatorname{Ker}\left(x_{t}\right)=\widetilde{C},
$$

and part (b) follows. Moreover, by Lemma 5.1, the induced action of $\Gamma / \operatorname{Ker}(\rho)$ on $V$ is generically free. By part (b), $\operatorname{Ker}(\rho)=\widetilde{C}$ and part (c) follows as well.

It remains to prove the claim. Choose $v_{i} \in V_{x_{i}}$ in general position and assume that $g=\left(g_{1}, \ldots, g_{r}\right)$ stabilizes $v:=\left(v_{1}, \ldots, v_{t}\right)$ in $V$ for some $g_{j} \in \mathrm{GL}_{p^{a_{j}}}$. Our goal is to show that $g_{j}$ is, in fact, central in $\mathrm{GL}_{p^{a_{j}}}$ for each $j=1, \ldots, r$.

Let us fix $j$ and focus on proving that $g_{j}$ is central for this particular $j$. By assumption (b) of Theorem 1.2, there exists an $i=1, \ldots, t$ such that $y_{i}$ is balanced and $y_{i j} \neq 0$. Let us assume that $y_{i j_{1}}, \ldots, y_{i j_{s}}= \pm 1$ and $y_{i h}=0$ for every $h \notin\left\{j_{1}, \ldots, j_{r}\right\}$ and consequently, $x_{i j_{1}}, \ldots, x_{i j_{s}}= \pm 1$ and $x_{i h}=0$ for every $h \notin\left\{j_{1}, \ldots, j_{r}\right\}$. By our assumption, $j \in\left\{j_{1}, \ldots, j_{s}\right\}$.

The representation $\rho_{x_{i}}$ of $\Gamma=\mathrm{GL}_{p^{a_{1}}} \times \cdots \times \mathrm{GL}_{p^{a_{r}}}$ on

$$
V_{x_{i}}:=V^{x_{i 1}} \otimes \cdots \otimes V^{x_{i t}}=V^{x_{i j_{1}}} \otimes \cdots \otimes V^{x_{i j_{s}}}
$$

factors through the projection $\Gamma \rightarrow \mathrm{GL}_{p^{a_{1}}} \times \cdots \times \mathrm{GL}_{p^{a_{j s}}}$. Thus if $g=\left(g_{1}, \ldots, g_{r}\right)$ stabilizes $v=\left(v_{1}, \ldots, v_{t}\right) \in V$ then, in particular, $g$ stabilizes $v_{i}$ and so $\left(g_{j_{1}}, \ldots, g_{j_{s}}\right)$ stabilizes $v_{i}$.

Since $y_{i}$ is assumed to be balanced, the conditions of Lemma 5.2 for the action of $\mathrm{GL}_{n_{j_{1}}} \times \cdots \times \mathrm{GL}_{n_{j_{s}}}$ on $V_{x_{i}}=V^{x_{j_{1}}} \otimes \cdots \otimes V^{x_{j_{s}}}$ are satisfied. (Recall that here $n_{i}=p^{a_{i}}$.) Since $\left(g_{j_{1}}, \ldots, g_{j_{s}}\right)$ stabilizes $v_{i} \in V_{x_{i}}$ in general position, Lemma 5.2 tells us that $g_{j_{1}}, \ldots, g_{j_{s}}$ are central in $\mathrm{GL}_{n_{j_{1}}}, \ldots, \mathrm{GL}_{n_{j_{s}}}$, respectively. In particular, $g_{j}$ is central in $\mathrm{GL}_{n_{j}}$, as desired. This completes the proof of Lemma 5.3 and thus of Theorem 1.2.

\section{Proof of Theorem 1.3}

Consider the central subgroups $\widetilde{C}$ and $C$ of $\Gamma=\mathrm{GL}_{p^{a_{1}}} \times \cdots \times \mathrm{GL}_{p^{a_{r}}}$ given by $\widetilde{C}=\left\{\left(\tau_{1}, \ldots, \tau_{r}\right) \in \mathbb{G}_{m}^{r} \mid \tau_{1} \cdots \tau_{r}=1\right\} \quad$ and $\quad C=\left\{\left(\tau_{1}, \ldots, \tau_{r}\right) \in \mu \mid \tau_{1} \cdots \tau_{r}=1\right\}$. Set $G:=\Gamma / C$ and $\widetilde{G}:=\Gamma / \widetilde{C}$. Note that $C=\widetilde{C} \cap \mu$. By Theorem A.1 and Corollary A.2, $H^{1}(-, G)$ and $H^{1}(-, \widetilde{G})$ are both isomorphic to the functor $\mathcal{F}$ defined in the statement of Theorem 1.3. In particular, $\operatorname{ed}(\widetilde{G})=\operatorname{ed}(G)=\operatorname{ed}(\mathcal{F})$ and $\operatorname{ed}_{p}(\widetilde{G})=\operatorname{ed}_{p}(G)=\operatorname{ed}_{p}(\mathcal{F})$. We are now ready to proceed with the proof of Theorem 1.3. 
(a) If $A_{1} \otimes \cdots \otimes A_{r}$ is split over $K$, then $A_{r}$ can be recovered from $A_{1}, \ldots, A_{r-1}$ as the unique central simple $K$-algebra of degree $p^{a_{r}}$ which is Brauer-equivalent to

$$
\left(A_{1} \otimes \cdots \otimes A_{r-1}\right)^{\mathrm{op}} \text {. }
$$

(Here $B^{\text {op }}$ denotes the opposite algebra of $B$.) In other words, the morphism of functors

$$
\mathcal{F} \rightarrow H^{1}\left(-, \mathrm{PGL}_{p^{a_{1}}}\right) \times \cdots \times H^{1}\left(-, \mathrm{PGL}_{p^{a_{r-1}}}\right)
$$

given by $\left(A_{1}, \ldots, A_{r-1}, A_{r}\right) \rightarrow\left(A_{1}, \ldots, A_{r-1}\right)$ is injective. We claim that if $a_{r} \geqslant a_{1}+\cdots+a_{r-1}$ (which is our assumption in part (a)), then this morphism is also surjective. Indeed,

$$
\operatorname{deg}\left(A_{1} \otimes \cdots \otimes A_{r-1}\right)=p^{a_{1}+\cdots+a_{r-1}}
$$

for any choice of central simple $K$-algebras $A_{1}, \ldots, A_{r-1}$ such that $\operatorname{deg}\left(A_{i}\right)=p^{a_{i}}$. Hence, for any such choice, there exists a central simple algebra of degree $p^{a_{r}}$ which is Brauer-equivalent to $\left(A_{1} \otimes \cdots \otimes A_{r-1}\right)^{\mathrm{op}}$. This proves the claim.

We conclude that if $a_{r} \geqslant a_{1}+\cdots+a_{r-1}$ then (20) is an isomorphism and thus

$$
\begin{gathered}
\operatorname{ed}(\widetilde{G})=\operatorname{ed}(G)=\operatorname{ed}(\mathcal{F})=\operatorname{ed}\left(\mathrm{PGL}_{p^{a_{1}}} \times \cdots \times \mathrm{PGL}_{p^{a_{r-1}}}\right), \\
\operatorname{ed}_{p}(\widetilde{G})=\operatorname{ed}_{p}(G)=\operatorname{ed}_{p}(\mathcal{F})=\operatorname{ed}_{p}\left(\mathrm{PGL}_{p^{a_{1}}} \times \cdots \times \mathrm{PGL}_{p^{a-1}}\right) .
\end{gathered}
$$

The inequality ed $(\mathcal{F}) \leqslant p^{2 a_{1}}+\cdots+p^{2 a_{r-1}}$ now follows from (9).

(b) Now suppose $a_{r}<a_{1}+\cdots+a_{r-1}$. Note that $\operatorname{Code}(C)$ has a minimal basis consisting of the single element $(1, \ldots, 1) \in\left(\mathbb{Z} / p^{a_{1}} \mathbb{Z}\right) \times \cdots \times\left(\mathbb{Z} / p^{a_{r}} \mathbb{Z}\right)$. Moreover, $p^{a_{r}} \leqslant \frac{1}{2} p^{a_{1}} \cdots p^{a_{r-1}}$ and consequently, Theorem 1.2 applies. It tells us that if the $r$-tuple $\left(p^{a_{1}}, \ldots, p^{a_{r}}\right)$ is not of the form $(2,2,2,2),(3,3,3)$ or $\left(2,2^{a}, 2^{a}\right)$, then

$$
\begin{aligned}
\operatorname{ed}(\mathcal{F})=\operatorname{ed}_{p}(\mathcal{F})=\operatorname{ed}(\tilde{G})=\operatorname{ed}_{p}(\widetilde{G}) & = \\
\operatorname{ed}(G) & =\operatorname{ed}_{p}(G)=p^{a_{1}+\cdots+a_{r}}-\sum_{i=1}^{r} p^{2 a_{i}}+r-1,
\end{aligned}
$$

as claimed.

(c) In the case where $\left(p^{a_{1}}, \ldots, p^{a_{r}}\right)=(2,2,2), \mathcal{F}(K)$ is the set of isomorphism classes of triples $\left(A_{1}, A_{2}, A_{3}\right)$ of quaternion $K$-algebras, such that $A_{1} \otimes A_{2} \otimes A_{3}$ is split over $K$. We will show that (i) $\operatorname{ed}(\mathcal{F}) \leqslant 3$ and (ii) $\operatorname{ed}_{2}(\mathcal{F}) \geqslant 3$.

To prove (i), recall that by a theorem of Albert [Lam 2005, Theorem III.4.8], the condition that $A_{1} \otimes A_{2} \otimes A_{3}$ is split over $K$ implies that $A_{1}$ and $A_{2}$ are linked over $K$. That is, there exist $a, b, c \in K^{*}$ such that $A_{1} \simeq(a, b)$ and $A_{2} \simeq(a, c)$ over $K$. Hence, the triple $\left(A_{1}, A_{2}, A_{3}\right) \in \mathcal{F}(K)$ descends to the triple $\left(B_{1}, B_{2}, B_{3}\right) \in \mathcal{F}\left(K_{0}\right)$, where $K_{0}=k(a, b, c), B_{1}=(a, b), B_{2}=(a, c)$ and $B_{3}=(a, b c)$ over $K_{0}$. Since $\operatorname{trdeg}\left(K_{0} / k\right) \leqslant 3$, assertion (i) follows. 
To prove (ii), consider the morphism of functors $f: \mathcal{F} \rightarrow H^{1}\left(-, \mathrm{SO}_{4}\right)$ given by

$$
f:\left(A_{1}, A_{2}, A_{3}\right) \mapsto \alpha,
$$

where $\alpha$ is a 4-dimensional quadratic form such that

$$
\alpha \oplus \mathbb{H} \oplus \mathbb{H} \cong N\left(A_{1}\right) \oplus\left(-N\left(A_{2}\right)\right) .
$$

Here $\mathbb{W}$ denotes the 2-dimensional hyperbolic form $\langle 1,-1\rangle, N\left(A_{1}\right)$ denotes the norm form of $A_{1}$, and $-N\left(A_{2}\right)$ denotes the opposite norm form of $A_{2}$, i.e., the unique 4-dimensional form such that $N\left(A_{2}\right) \oplus\left(-N\left(A_{2}\right)\right)$ is hyperbolic. Since $N\left(A_{1}\right)$ and $N\left(A_{2}\right)$ are forms of discriminant 1 , so is $\alpha$ (this will also be apparent from the explicit computations below). Thus we may view $\alpha$ as an element of the Galois cohomology set $H^{1}\left(K, \mathrm{SO}_{4}\right)$, which classifies 4-dimensional quadratic forms of discriminant 1 over $K$, up to isomorphism. Note also that by the Witt cancellation theorem, $\alpha$ is unique up to isomorphism. We conclude that the morphism of functors $f$ is well-defined.

Equivalently, using the definition of the Albert form given in [Lam 2005, p. 69], $\alpha$ is the unique 4-dimensional quadratic form such that $\alpha \oplus \mathbb{H} \cong q$, where $q$ is the 6-dimensional Albert form of $A_{1}$ and $A_{2}$. Here the Albert form of $A_{1}$ and $A_{2}$ is isotropic, and hence, can be written as $\alpha \oplus \mathbb{H}$, because $A_{1}$ and $A_{2}$ are linked; once again, see [Lam 2005, Theorem III.4.8].

Suppose $A_{1}=(a, b), A_{2}=(a, c)$, and $A_{3}=(a, b c)$, as above. Then

$$
N\left(A_{1}\right)=\langle\langle-a,-b\rangle\rangle=\langle 1,-a,-b, a b\rangle,
$$

and similarly $N\left(A_{2}\right)=\langle 1,-a,-c, a c\rangle$; see, e.g., [Lam 2005, Corollary III.2.2]. Thus

$N\left(A_{1}\right) \oplus\left(-N\left(A_{2}\right)\right)=\langle 1,-1,-a, a,-b, c, a b,-a c\rangle \simeq\langle-b, c, a b,-a c\rangle \oplus \mathbb{H} \oplus \mathbb{H}$, and we obtain an explicit formula for $\alpha=f\left(A_{1}, A_{2}, A_{3}\right): \alpha \cong\langle-b, c, a b,-a c\rangle$.

It is easy to see that any 4-dimensional quadratic form of discriminant 1 over $K$ can be written as $\langle-b, c, a b,-a c\rangle$ for some $a, b, c \in K^{*}$. In other words, the morphism of functors $f: \mathcal{F} \rightarrow H^{1}\left(-, \mathrm{SO}_{4}\right)$ is surjective. Consequently,

$$
\operatorname{ed}_{2}(\mathcal{F}) \geq \operatorname{ed}_{2}\left(H^{1}\left(-, \mathrm{SO}_{4}\right)\right)=\operatorname{ed}_{2}\left(\mathrm{SO}_{4}\right)
$$

see, e.g., [Berhuy and Favi 2003, Lemma 1.9] or [Reichstein 2010, Lemma 2.2]. On the other hand, $\mathrm{ed}_{2}\left(\mathrm{SO}_{4}\right)=3$; see [Reichstein and Youssin 2000, Theorem 8.1(2) and Remark 8.2] or [Reichstein 2010, Corollary 3.6(a)]. Thus

$$
\operatorname{ed}_{2}(\mathcal{F}) \geqslant \operatorname{ed}_{2}\left(\mathrm{SO}_{4}\right)=3 \text {. }
$$

This completes the proof of (ii) and thus of part (c) of Theorem 1.3. 


\section{Appendix: Galois cohomology of central quotients of products of general linear groups}

Athena Nguyen $\mathbf{1}$

In this appendix we will study the Galois cohomology of algebraic groups of the form

$$
G:=\Gamma / C
$$

where $\Gamma:=\mathrm{GL}_{n_{1}} \times \cdots \times \mathrm{GL}_{n_{r}}$ and $C \subset Z(\Gamma)=\mathbb{G}_{m}^{r}$ is a central subgroup. Here $n_{1}, \ldots, n_{r} \geqslant 1$ are integers, not necessarily prime powers. Let

$$
\bar{G}:=G / Z(G)=\mathrm{PGL}_{n_{1}} \times \cdots \times \mathrm{PGL}_{n_{r}}=\Gamma / Z(\Gamma) .
$$

Recall that for any field $K / k, H^{1}\left(K, \mathrm{PGL}_{n}\right)$ is naturally identified with the set of isomorphism classes of central simple $K$-algebras of degree $n$, and

$$
H^{1}(K, \bar{G})=H^{1}\left(K, \mathrm{PGL}_{n_{1}}\right) \times \cdots \times H^{1}\left(K, \mathrm{PGL}_{n_{r}}\right)
$$

is identified with the set of $r$-tuples $\left(A_{1}, \ldots, A_{r}\right)$ of central simple $K$-algebras such that $\operatorname{deg}\left(A_{i}\right)=n_{i}$. Denote by $\partial_{K}^{i}$ the coboundary map $H^{1}\left(K, \mathrm{PGL}_{n_{i}}\right) \rightarrow$ $H^{2}\left(K, \mathbb{G}_{m}\right)$ induced by the short exact sequence

$$
1 \rightarrow \mathbb{G}_{m} \rightarrow \mathrm{GL}_{n_{i}} \rightarrow \mathrm{PGL}_{n_{i}} \rightarrow 1 .
$$

This map sends a central simple algebra $A_{i}$ to its Brauer class $\left[A_{i}\right]$ in $H^{2}\left(K, \mathbb{G}_{m}\right)=$ $\operatorname{Br}(K)$.

Of particular interest to us will be

$$
X\left(\mathbb{G}_{m}^{r} / C\right)=\left\{\left(m_{1}, \ldots, m_{r}\right) \in \mathbb{Z}^{r} \mid \tau_{1}^{m_{1}} \cdots \tau_{r}^{m_{r}}=1 \quad \forall\left(\tau_{1}, \ldots, \tau_{r}\right) \in \mathbb{G}_{m}^{r}\right\},
$$

as in (6). We are now ready to state the main result of this appendix.

Theorem A.1. Let $\pi: G \rightarrow \bar{G}:=\mathrm{PGL}_{n_{1}} \times \cdots \times \mathrm{PGL}_{n_{r}}$ be the natural projection and $\pi_{*}: H^{1}(K, G) \rightarrow H^{1}(K, \bar{G})$ be the induced map in cohomology. Here $K / k$ is a field extension. Then:

(a) The map $\pi_{*}: H^{1}(K, G) \rightarrow H^{1}(K, \bar{G})$ is injective for every field $K / k$.

(b) The map $\pi_{*}$ identifies $H^{1}(K, G)$ with the set of isomorphism classes of $r$-tuples $\left(A_{1}, \ldots, A_{r}\right)$ of central simple $K$-algebras such that $\operatorname{deg}\left(A_{i}\right)=n_{i}$ and $A_{1}^{\otimes m_{1}} \otimes \cdots \otimes A_{r}^{\otimes m_{r}}$ is split over $K$ for every $\left(m_{1}, \ldots, m_{r}\right) \in X\left(\mathbb{G}_{m}^{r} / C\right)$.

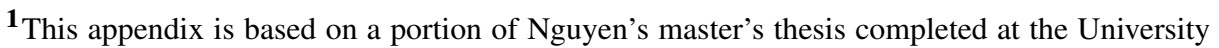
of British Columbia. Nguyen gratefully acknowledges the financial support from the University of British Columbia and the Natural Sciences and Engineering Research Council of Canada. 
Proof. Throughout, we will identify $H^{2}\left(K, \mathbb{G}_{m}^{r}\right)$ with $H^{2}\left(K, \mathbb{G}_{m}\right)^{r}$ and $X\left(\mathbb{G}_{m}^{r}\right)$ with $\mathbb{Z}^{n}$. A character $x=\left(m_{1}, \ldots, m_{r}\right) \in \mathbb{Z}^{n}$, i.e., a character $x: \mathbb{G}_{m}^{r} \rightarrow \mathbb{G}_{m}$ given by $\left(\tau_{1}, \ldots, \tau_{r}\right) \rightarrow \tau_{1}^{m_{1}} \cdots \tau_{r}^{m_{r}}$, induces a map $x_{*}: H^{2}\left(K, \mathbb{G}_{m}\right)^{r} \rightarrow H^{2}\left(K, \mathbb{G}_{m}\right)$ in cohomology given by

$$
x_{*}\left(\alpha_{1}, \ldots, \alpha_{r}\right)=\alpha_{1}^{m_{1}} \cdots \cdots \alpha_{r}^{m_{r}} .
$$

Let us now consider the diagram

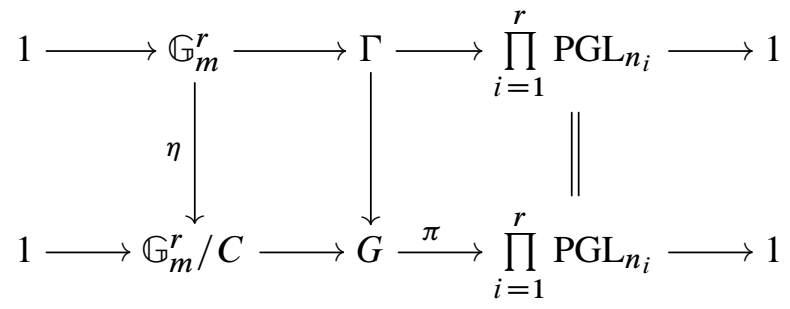

Since $H^{1}\left(K, \mathbb{G}_{m}^{r} / C\right)=\{1\}$ by Hilbert's theorem 90, we obtain the following diagram in cohomology with exact rows:

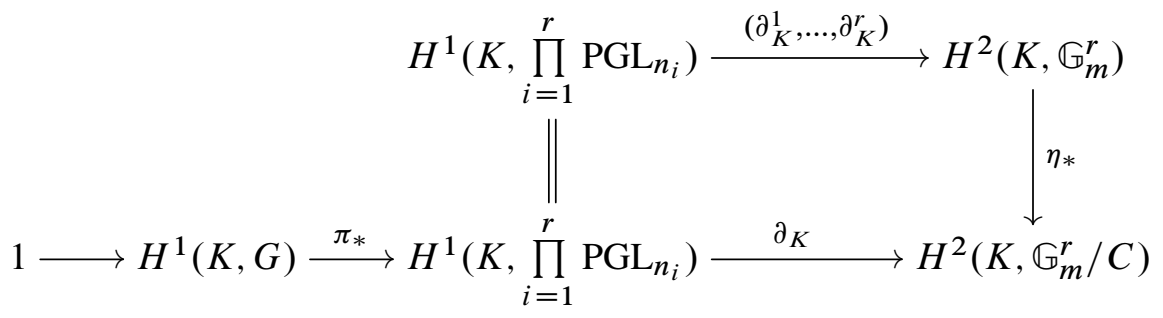

(a) It follows from [Serre 1997, I.5, Proposition 42] that $\pi_{*}$ is injective.

(b) Thus, $\pi_{*}$ identifies $H^{1}(K, G)$ with the set of $r$-tuples $\left(A_{1}, \ldots, A_{r}\right)$, where $A_{i} \in$ $H^{1}\left(K, \mathrm{PGL}_{n_{i}}\right)$ is a central simple algebra of degree $n_{i}$, and $\left(\partial_{K}^{1}\left(A_{1}\right), \ldots, \partial_{K}^{r}\left(A_{r}\right)\right) \in$ $\operatorname{Ker}\left(\eta_{*}\right)$. Recall that $\partial_{K}^{i}$ sends a central simple algebra $A_{i}$ to its Brauer class $\left[A_{i}\right] \in H^{2}\left(K, \mathbb{G}_{m}\right)$. In the sequel we will use additive notation for the abelian group $H^{2}\left(K, \mathbb{G}_{m}\right)=\operatorname{Br}(K)$.

Consider an $r$-tuple $\alpha:=\left(\left[A_{1}\right], \ldots,\left[A_{r}\right]\right) \in H^{2}\left(K, \mathbb{G}_{m}^{r}\right)$. Since $\mathbb{G}_{m}^{r} / C$ is diagonalizable, $\eta_{*}(\alpha)=0$ if and only if $x_{*}\left(\eta_{*}(\alpha)\right)=0$ for all $x \in X\left(\mathbb{G}_{m}^{r} / C\right)$. If $x=\left(m_{1}, \ldots, m_{r}\right) \in X\left(\mathbb{G}_{m}^{r} / C\right)$, then $x_{*} \circ \eta_{*}=\left(m_{1}, \ldots, m_{r}\right) \in X\left(G_{m}^{r}\right)$. By (21), $x_{*}\left(\eta_{*}(\alpha)\right)=\left[A_{1}^{\otimes m_{1}} \otimes \cdots \otimes A_{r}^{\otimes m_{r}}\right]$, and part (b) follows.

Corollary A.2. Let $\Gamma:=\mathrm{GL}_{n_{1}} \times \cdots \times \mathrm{GL}_{n_{r}}, C_{1}, C_{2}$ be $k$-subgroups of $Z(\Gamma)=\mathbb{G}_{m}^{r}$, $G_{1}=\Gamma / C_{1}$ and $G_{2}=\Gamma / C_{2}$. Denote the central subgroup $\mu_{n_{1}} \times \cdots \times \mu_{n_{r}}$ of $\Gamma$ by $\mu$.

If $C_{1} \cap \mu=C_{2} \cap \mu$ then the Galois cohomology functors $H^{1}\left(-, G_{1}\right)$ and $H^{1}\left(-, G_{2}\right)$ are isomorphic. 
Proof. By Theorem A.1, $H^{1}\left(K, G_{i}\right)$ is naturally identified with the set of $r$-tuples $\left(A_{1}, \ldots, A_{r}\right)$ of central simple algebras such that $\operatorname{deg}\left(A_{i}\right)=n_{i}$ and

$A_{1}^{\otimes m_{1}} \otimes \cdots \otimes A_{r}^{\otimes m_{r}}$ is split over $K$ for every $\left(m_{1}, \ldots, m_{r}\right) \in X\left(\mathbb{G}_{m} / C_{i}\right)$.

Note that since $A_{i}^{\otimes n_{i}}$ is split for every $i$, this condition depends only on the image of $\left(m_{1}, \ldots, m_{r}\right)$ under the natural projection

$$
\pi: X\left(\mathbb{G}_{m}^{r}\right)=\mathbb{Z}^{r} \rightarrow\left(\mathbb{Z} / n_{1} \mathbb{Z}\right) \times \cdots \times\left(\mathbb{Z} / n_{r} \mathbb{Z}\right)=X(\mu) .
$$

Our assumption that $C_{1} \cap \mu=C_{2} \cap \mu$ is equivalent to $X\left(\mathbb{G}_{m}^{r} / C_{1}\right)$ and $X\left(\mathbb{G}_{m}^{r} / C_{2}\right)$ having the same image under $\pi$, and the corollary follows.

In order to state the second corollary of Theorem A.1, we will need the following definition. By a code we shall mean a subgroup of $X(\mu)=\left(\mathbb{Z} / n_{1} \mathbb{Z}\right) \times \cdots \times\left(\mathbb{Z} / n_{r} \mathbb{Z}\right)$. Given a subgroup $C \subset \mu$, we define the code $\operatorname{Code}(C):=X(\mu / C)$, as in (8).

We will say that two codes are called equivalent if one can be obtained from the other by repeatedly performing the following elementary operations:

(1) Permuting entries $i$ and $j$ in every vector of the code, for any $i, j$ with $n_{i}=n_{j}$.

(2) Multiplying the $i$-th entry in every vector of the code by an integer $c$ prime to $n_{i}$.

Corollary A.3. Suppose $C_{1}$ and $C_{2}$ are subgroups of $\mu:=\mu_{n_{1}} \times \cdots \times \mu_{n_{r}}, G_{1}=$ $\Gamma / C_{1}$ and $G_{2}:=\Gamma / C_{2}$. If $\operatorname{Code}\left(C_{1}\right)$ and $\operatorname{Code}\left(C_{2}\right)$ are equivalent, then

(a) the Galois cohomology functors $H^{1}\left(-, G_{1}\right), H^{1}\left(-, G_{2}\right)$ are isomorphic, and (b) in particular, $\operatorname{ed}\left(G_{1}\right)=\operatorname{ed}\left(G_{2}\right)$ and $\operatorname{ed}_{p}\left(G_{1}\right)=\operatorname{ed}_{p}\left(G_{2}\right)$ for every prime $p$.

Proof. To prove part (a), it suffices to show that $H^{1}\left(-, G_{1}\right)$ and $H^{1}\left(-, G_{2}\right)$ are isomorphic if $C_{2}$ is obtained from $C_{1}$ by an elementary operation.

(1) Suppose $n_{i}=n_{j}$ for some $i, j=1, \ldots, r$, and $\operatorname{Code}\left(C_{2}\right)$ is obtained from $\operatorname{Code}\left(C_{1}\right)$ by permuting entries $i$ and $j$ in every vector. In this case $C_{2}=\alpha\left(C_{1}\right)$, where $\alpha$ is the automorphism of $\Gamma=\mathrm{GL}_{n_{1}} \times \cdots \times \mathrm{GL}_{n_{r}}$ which swaps the $i$-th and the $j$-th components. Then $\alpha$ induces an isomorphism between $G_{1}=\Gamma / C_{1}$ and $G_{2}=\Gamma / C_{2}$, and thus an isomorphism between $H^{1}\left(-, G_{1}\right)$ and $H^{1}\left(-, G_{2}\right)$.

(2) Now suppose that $\operatorname{Code}\left(C_{1}\right)$ is obtained from $\operatorname{Code}\left(C_{2}\right)$ by multiplying the $i$-th entry in every vector by some $c \in\left(\mathbb{Z} / n_{i} \mathbb{Z}\right)^{*}$. The description of $H^{1}(K, G / \mu)$ given by Theorem A.1 now tells us that

$$
\begin{aligned}
H^{1}\left(K, G_{1}\right) & \rightarrow H^{1}\left(K, G_{2}\right), \\
\left(A_{1}, \ldots, A_{r}\right) & \mapsto\left(A_{1}, \ldots, A_{i-1},\left[A_{i}^{\otimes c}\right]_{n_{i}}, A_{i+1}, \ldots, A_{r}\right),
\end{aligned}
$$

is an isomorphism. Here, by $\left[A_{i}^{\otimes c}\right]_{n_{i}}$ we mean the unique central simple $K$-algebra of degree $n_{i}$ which is Brauer equivalent to $A_{i}^{\otimes c}$.

Part (b) follows from (a), because $\operatorname{ed}(G)$ and $\operatorname{ed}_{p}(G)$ are defined entirely in terms of the Galois cohomology functor $H^{1}(-, G)$. 


\section{Acknowledgement}

The authors are grateful to the anonymous referee for a careful reading of this paper and for numerous helpful comments.

\section{References}

[Auel et al. 2011] A. Auel, E. Brussel, S. Garibaldi, and U. Vishne, "Open problems on central simple algebras”, Transform. Groups 16:1 (2011), 219-264. MR 2012e:16050 Zbl 1230.16016

[Baek and Merkurjev 2012] S. Baek and A. S. Merkurjev, "Essential dimension of central simple algebras”, Acta Math. 209:1 (2012), 1-27. MR 2979508 Zbl 1258.16023

[Berhuy and Favi 2003] G. Berhuy and G. Favi, "Essential dimension: a functorial point of view (after A. Merkurjev)”, Doc. Math. 8 (2003), 279-330. MR 2004m:11056 Zbl 1101.14324

[Brosnan et al. 2011] P. Brosnan, Z. Reichstein, and A. Vistoli, "Essential dimension of moduli of curves and other algebraic stacks”, J. Eur. Math. Soc. (JEMS) 13:4 (2011), 1079-1112. MR 2012g:14012 Zbl 1234.14003

[Karpenko and Merkurjev 2008] N. A. Karpenko and A. S. Merkurjev, "Essential dimension of finite p-groups", Invent. Math. 172:3 (2008), 491-508. MR 2009b:12009 Zbl 1200.12002

[Lam 2005] T. Y. Lam, Introduction to quadratic forms over fields, Graduate Studies in Mathematics 67, American Mathematical Society, Providence, RI, 2005. MR 2005h:11075 Zbl 1068.11023

[Lang 2002] S. Lang, Algebra, 3rd ed., Graduate Texts in Mathematics 211, Springer, New York, 2002. MR 2003e:00003 Zbl 0984.00001

[Lemire 2004] N. Lemire, "Essential dimension of algebraic groups and integral representations of Weyl groups", Transform. Groups 9:4 (2004), 337-379. MR 2005j:20056 Zbl 1076.14060

[Lorenz et al. 2003] M. Lorenz, Z. Reichstein, L. H. Rowen, and D. J. Saltman, "Fields of definition for division algebras", J. London Math. Soc. (2) 68:3 (2003), 651-670. MR 2004j:16022 Zbl 1071.16012

[Lötscher 2013] R. Lötscher, "A fiber dimension theorem for essential and canonical dimension", Compos. Math. 149:1 (2013), 148-174. MR 3011881 Zbl 1260.14059

[Lötscher et al. 2013] R. Lötscher, M. MacDonald, A. Meyer, and Z. Reichstein, "Essential dimension of algebraic tori”, J. Reine Angew. Math. 677 (2013), 1-13. MR 3039772 Zbl 06162480

[MacWilliams and Sloane 1977] F. J. MacWilliams and N. J. A. Sloane, The theory of error-correcting codes, I, North-Holland Mathematical Library 16, North-Holland, Amsterdam, 1977. MR 57 \#5408a Zbl 0369.94008

[Merkurjev 2013] A. S. Merkurjev, "Essential dimension: a survey", Transform. Groups 18:2 (2013), 415-481. MR 3055773 Zbl 1278.14066

[Popov 1987] A. M. Popov, "Finite isotropy subgroups in general position of irreducible semisimple linear Lie groups", Trudy Moskov. Mat. Obshch. 50 (1987), 209-248, 262. In Russian; translated in Trans. Moscow Math. Soc. (1988), 205-249. MR 89a:20049 Zbl 0661.22009

[Popov and Vinberg 1994] V. L. Popov and E. B. Vinberg, "Invariant theory", pp. 123-278 in Algebraic geometry, IV, edited by I. R. Shafarevich and A. N. Parshin, Encyclopaedia of Mathematical Sciences 55, Springer, Berlin, 1994. MR 95g:14002 Zbl 0796.14002

[Procesi 1967] C. Procesi, "Non-commutative affine rings", Atti Accad. Naz. Lincei Mem. Cl. Sci. Fis. Mat. Natur. Sez. I (8) 8 (1967), 237-255. MR 37 \#256 Zbl 0204.04802

[Reichstein 1999] Z. Reichstein, "On a theorem of Hermite and Joubert", Canad. J. Math. 51:1 (1999), 69-95. MR 2000h:12008 Zbl 0942.12001 
[Reichstein 2010] Z. Reichstein, "Essential dimension", pp. 162-188 in Proceedings of the International Congress of Mathematicians, volume II, Hindustan Book Agency, New Delhi, 2010. MR 2012g:11074 Zbl 1232.14030

[Reichstein 2012] Z. Reichstein, "What is. . essential dimension?", Notices Amer. Math. Soc. 59:10 (2012), 1432-1434. MR 3025902 Zbl 1284.12003

[Reichstein and Vonessen 2007] Z. Reichstein and N. Vonessen, "Tame group actions on central simple algebras", J. Algebra 318:2 (2007), 1039-1056. MR 2008j:16061 Zbl 1137.16025

[Reichstein and Youssin 2000] Z. Reichstein and B. Youssin, "Essential dimensions of algebraic groups and a resolution theorem for G-varieties", Canad. J. Math. 52 (2000), 1018-1056. MR 2001k: 14088 Zbl 1044.14023

[Richardson 1972] R. W. Richardson, Jr., "Principal orbit types for algebraic transformation spaces in characteristic zero", Invent. Math. 16 (1972), 6-14. MR 45 \#3405 Zbl 0242.14010

[Serre 1997] J.-P. Serre, Galois cohomology, Springer, Berlin, 1997. MR 98g:12007 Zbl 0902.12004

[Shimura 1971] G. Shimura, Introduction to the arithmetic theory of automorphic functions, Publications of the Mathematical Society of Japan 11, Princeton University Press, 1971. MR 47 \#3318 Zbl 0221.10029

Received November 27, 2014. Revised March 25, 2015.

\section{Shane Cernele}

DEPARTMENT OF MATHEMATICS

UNIVERSITY OF BRITISH COLUMBIA

1984 MATHEMATICS ROAD

VANCOUVER BC V6T1Z2

CANADA

scernele@math.ubc.ca

ZINOVY REICHSTEIN

DEPARTMENT OF MATHEMATICS

UNIVERSITY OF BRITISH COLUMBIA

1984 MATHEMATICS ROAD

VANCOUVER BC V6T1Z2

CANADA

reichst@math.ubc.ca

ATHENA NGUYEN

DEPARTMENT OF MATHEMATICS

UNIVERSITY OF BRITISH COLUMBIA

1984 MATHEMATICS ROAD

VANCOUVER BC V6T1Z2

CANADA

athena@math.ubc.ca 


\title{
PACIFIC JOURNAL OF MATHEMATICS
}

\author{
msp.org/pjm
}

Founded in 1951 by E. F. Beckenbach (1906-1982) and F. Wolf (1904-1989)

\section{EDITORS}

Don Blasius (Managing Editor)

Department of Mathematics

University of California

Los Angeles, CA 90095-1555

blasius@math.ucla.edu

\author{
Paul Balmer \\ Department of Mathematics \\ University of California \\ Los Angeles, CA 90095-1555 \\ balmer@math.ucla.edu \\ Robert Finn \\ Department of Mathematics \\ Stanford University \\ Stanford, CA 94305-2125 \\ finn@math.stanford.edu \\ Sorin Popa \\ Department of Mathematics \\ University of California \\ Los Angeles, CA 90095-1555 \\ popa@math.ucla.edu
}

\author{
Vyjayanthi Chari \\ Department of Mathematics \\ University of California \\ Riverside, CA 92521-0135 \\ chari@math.ucr.edu \\ Kefeng Liu \\ Department of Mathematics \\ University of California \\ Los Angeles, CA 90095-1555 \\ liu@math.ucla.edu \\ Jie Qing \\ Department of Mathematics \\ University of California \\ Santa Cruz, CA 95064 \\ qing@ cats.ucsc.edu
}

\section{PRODUCTION}

Silvio Levy, Scientific Editor, production@msp.org

\section{SUPPORTING INSTITUTIONS}

ACADEMIA SINICA, TAIPEI

CALIFORNIA INST. OF TECHNOLOGY

INST. DE MATEMÁTICA PURA E APLICADA

KEIO UNIVERSITY

MATH. SCIENCES RESEARCH INSTITUTE

NEW MEXICO STATE UNIV.

OREGON STATE UNIV.

\author{
STANFORD UNIVERSITY \\ UNIV. OF BRITISH COLUMBIA \\ UNIV. OF CALIFORNIA, BERKELEY \\ UNIV. OF CALIFORNIA, DAVIS \\ UNIV. OF CALIFORNIA, LOS ANGELES \\ UNIV. OF CALIFORNIA, RIVERSIDE \\ UNIV. OF CALIFORNIA, SAN DIEGO \\ UNIV. OF CALIF., SANTA BARBARA
}

\author{
Daryl Cooper \\ Department of Mathematics \\ University of California \\ Santa Barbara, CA 93106-3080 \\ cooper@math.ucsb.edu \\ Jiang-Hua Lu \\ Department of Mathematics \\ The University of Hong Kong \\ Pokfulam Rd., Hong Kong \\ jhlu@maths.hku.hk \\ Paul Yang \\ Department of Mathematics \\ Princeton University \\ Princeton NJ 08544-1000 \\ yang@math.princeton.edu
}

These supporting institutions contribute to the cost of publication of this Journal, but they are not owners or publishers and have no responsibility for its contents or policies.

See inside back cover or msp.org/pjm for submission instructions.

The subscription price for 2015 is US \$420/year for the electronic version, and \$570/year for print and electronic.

Subscriptions, requests for back issues and changes of subscribers address should be sent to Pacific Journal of Mathematics, P.O. Box 4163, Berkeley, CA 94704-0163, U.S.A. The Pacific Journal of Mathematics is indexed by Mathematical Reviews, Zentralblatt MATH, PASCAL CNRS Index, Referativnyi Zhurnal, Current Mathematical Publications and Web of Knowledge (Science Citation Index).

The Pacific Journal of Mathematics (ISSN 0030-8730) at the University of California, c/o Department of Mathematics, 798 Evans Hall \#3840, Berkeley, CA 94720-3840, is published twelve times a year. Periodical rate postage paid at Berkeley, CA 94704, and additional mailing offices. POSTMASTER: send address changes to Pacific Journal of Mathematics, P.O. Box 4163, Berkeley, CA 94704-0163.

PJM peer review and production are managed by EditFLOW ${ }^{\circledR}$ from Mathematical Sciences Publishers.

\section{PUBLISHED BY}

\section{mathematical sciences publishers \\ nonprofit scientific publishing}

http://msp.org/

(C) 2015 Mathematical Sciences Publishers 


\title{
PACIFIC JOURNAL OF MATHEMATICS
}

\author{
Volume 279 No. 1-2 December 2015
}

In memoriam: Robert Steinberg

Robert Steinberg (1922-2014): In memoriam V. S. VARADARAJAN

Cellularity of certain quantum endomorphism algebras

HENNING H. ANDERSEN, GUSTAV I. LEHRER and RUIBIN ZHANG

Lower bounds for essential dimensions in characteristic 2 via orthogonal representations ANTONIO BABIC and VLADIMIR CHERNOUSOV

Cocharacter-closure and spherical buildings

Michael Bate, Sebastian Herpel, Benjamin Martin and Gerhard RöHrLe

Embedding functor for classical groups and Brauer-Manin obstruction

Eva Bayer-Fluckiger, Ting-Yu LeE and Raman Parimala

On maximal tori of algebraic groups of type $G_{2}$

Constantin Beli, PhilipPe Gille and Ting-Yu LeE

On extensions of algebraic groups with finite quotient

MICHEL BRION

Essential dimension and error-correcting codes

SHANE CERNELE and ZiNOVy REICHSTEIN

Notes on the structure constants of Hecke algebras of induced representations of finite Chevalley groups

Charles W. CuRTis

Complements on disconnected reductive groups

FRANÇOIS DIGNE and JEAN MICHEL

Extending Hecke endomorphism algebras

Jie Du, Brian J. Parshall and LeOnard L. SCOTT

Products of partial normal subgroups

ELLEN HENKE

Lusztig induction and $\ell$-blocks of finite reductive groups

RADHA KESSAR and GUNTER MALLE

Free resolutions of some Schubert singularities

Manoj Kummini, Venkatramani Lakshmibai, Pramathanath Sastry and C. S. Seshadri

Free resolutions of some Schubert singularities in the Lagrangian Grassmannian

VenKatramani LAKSHMibai and ReUVEN HODGES

Distinguished unipotent elements and multiplicity-free subgroups of simple algebraic groups

Martin W. Liebeck, Gary M. Seitz and Donna M. Testerman

Action of longest element on a Hecke algebra cell module

GEORGE LUSZTIG

Generic stabilisers for actions of reductive groups

BENJAMIN MARTIN

On the equations defining affine algebraic groups

VLADIMIR L. POPOV

Smooth representations and Hecke modules in characteristic $p$

PETER SCHNEIDER

On CRDAHA and finite general linear and unitary groups

BHAMA SRINIVASAN

Weil representations of finite general linear groups and finite special linear groups PHAM HUU TIEP

The pro- $p$ Iwahori Hecke algebra of a reductive $p$-adic group, $\mathrm{V}$ (parabolic induction) MARIE-FRANCE VIGNÉRAS

Acknowledgement 